\title{
Wind-structure interaction simulations of ovalling vibrations in silo groups
}

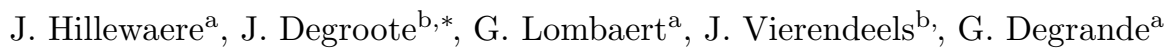 \\ ${ }^{a}$ Department of Civil Engineering, $K U$ Leuven, \\ Kasteelpark Arenberg 40, BE-3001 Heverlee, Belgium \\ ${ }^{b}$ Department of Flow, Heat and Combustion Mechanics, Ghent University, \\ Sint-Pietersnieuwstraat 41, BE-9000 Ghent, Belgium
}

\begin{abstract}
During a storm in October 2002, wind-induced ovalling vibrations were observed on several empty silos of a closely spaced group of silos in the port of Antwerp (Belgium). In this paper, three-dimensional numerical simulations are used to investigate this complex case of wind-structure interaction. The computed amplitude of the ovalling vibrations of the silos is similar to that in the observations, indicating that the adopted modelling approach can be suitable for the analysis of new silo groups.

Both one-way and two-way simulations are presented, for a single silo and for the silo group. In the one-way simulations, the wind pressure is applied on the structure, disregarding the structural displacements in the wind flow simulation. By contrast, the two-way simulations also take into account the effect of the structural motion on the wind flow. For a single silo, the one-way and two-way simulations yield similar results. Conversely, for a silo in the group, the ovalling vibrations are significantly larger in the two-way simulations than in the one-way simulations. Consequently, aeroelastic effects and/or interactions between the wake-induced excitation and the vibration are present in the silo group for the investigated case.
\end{abstract}

\footnotetext{
${ }^{*}$ Corresponding author

Email addresses: jeroen.hillewaere@bwk.kuleuven.be (J. Hillewaere), joris.degroote@ugent.be (J. Degroote), geert.lombaert@bwk.kuleuven.be (G. Lombaert), jan.vierendeels@ugent.be (J. Vierendeels), geert.degrande@bwk.kuleuven.be (G. Degrande)
}

Preprint submitted to Journal of Fluids and Structures 
Furthermore, it is shown that the aerodynamic loading and vibration amplitudes are considerably larger for silos in the group than for a single isolated silo.

Keywords: wind-structure interaction, aeroelastic instability, wind-induced vibration, ovalling, silo

\section{Introduction}

Given the tendency to build ever taller, more slender and hence increasingly flexible structures, many present-day constructions are susceptible to windinduced vibrations. Although mostly very small, these vibrations can become excessive and there are many examples of catastrophic structural failure due to wind-induced vibrations (Païdoussis et al., 2011; Belloli et al., 2011; Shellard, 1967; Billah and Scanlan, 1991). For the design of flexible structures, it is therefore increasingly important to understand the mechanisms that are causing these vibrations and to provide engineers with proper methodologies and tools to investigate these phenomena.

In the present paper, the case of a group of 40 silos in the port of Antwerp (Belgium) is studied (figure 1). During a storm in 2002 wind-induced ovalling vibrations with an amplitude of approximately $0.1 \mathrm{~m}$ have been observed on several empty silos at the windward side of the group. This vibration is significant compared to the gaps of $0.3 \mathrm{~m}$ between the silos. As is typical for ovalling deformations, the cross section of the axisymmetrical structure deforms as a shell, without bending deformation with respect to the longitudinal axis of symmetry (Païdoussis and Wong, 1982).

The ovalling vibrations of the silo group show some similarity with the collapse of 3 cooling towers from a group of 8 at Ferrybridge (UK) during a gale in 1965 (Pope, 1994). However, these collapsed cooling towers were located at the leeward side of the group while the ovalling vibrations in the silo group occurred on the windward side. A major difference between the silo group and the cooling towers is the size of the gaps between the individual objects, respectively $5 \%$ 


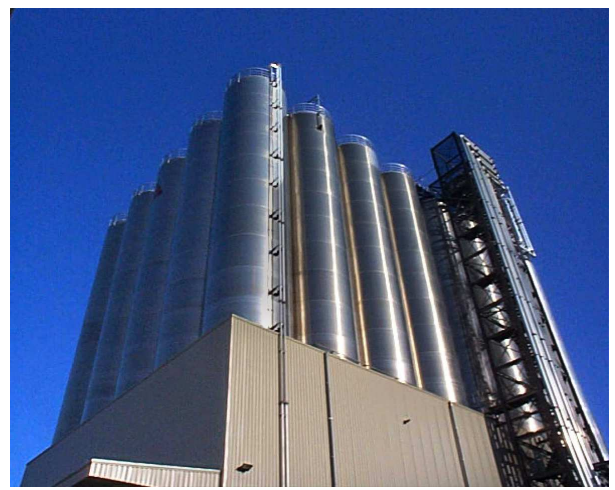

Figure 1: Southwest corner of the silo group in the port of Antwerp (Belgium) where ovalling vibrations were observed in October 2002.

of the diameter compared to approximately one diameter. This entails that the flow blockage effect for the silo group is much higher than for the cooling towers. Furthermore, the cooling towers are made of reinforced concrete as opposed to aluminium for the silo group, resulting in lower damping for the latter. The investigation of this incident with the cooling towers identified a tensile meridional failure within the shell fabric as dominant initial mode of failure. For the particular wind direction during the gale, the mean meridional force increased by $30 \%$ and the resonance force doubled, whereas the quasi-static fluctuating force remained more or less the same compared to an isolated tower (Pope, 1994).

Because of the complexity of this wind-structure interaction (WSI) problem, a simplified phenomenological model or experimental methods are not suitable to investigate the ovalling vibrations. Other aeroelastic applications, e.g. flutter of bridge decks or galloping of cables can be modelled with a two degrees of freedom system in a wind tunnel (Païdoussis et al., 2011). However, a scale model of a silo structure that has the appropriate flexibility is not easily constructed. Furthermore, in situ measurements of the structural response under normal wind loading by Dooms et al. (2006) could not decisively pinpoint what mechanism is causing the ovalling vibrations. 
By contrast, the versatility of numerical techniques to simultaneously incorporate complex wind flow details and structural flexibility, even for problems with complex geometries, is a great advantage. Procedures are available to couple a numerical model for the wind flow, i.e. computational fluid dynamics (CFD), and the structure, e.g. finite element (FE) models. Hence, this WSI problem can be studied numerically. Numerical studies on the flutter of bridges have been performed by Mannini et al. (2011a) and Šarkić et al. (2012), for example. Furthermore, the motion of light-weight membrane structures under wind load is analyzed numerically by Hojjat et al. (2010) and Michalski et al. (2011). In addition, the deflection of wind turbine blades during operation is calculated by Bazilevs et al. (2011). However, the behaviour of silos and other cylinders under wind loading is still often investigated without taking the structural displacement into account in the flow calculation (Uernatsu et al., 2015; Gorski et al., 2015; Zhao et al., 2013, 2014).

In this paper, WSI simulations are performed to investigate the wind-induced ovalling vibrations of individual silos mounted in a group arrangement. Compared to our previous work related to this silo group (Hillewaere et al., 2012), a significantly different methodology is proposed. Our previous work only contained CFD simulations, with rigid structures. In this work, flexible structures are considered, with and without including the influence of their motion on the wind flow. The model of the silo group was $2 \mathrm{D}$ in our previous work and has been extended to 3D in this paper, which among other things entails including the atmospheric boundary layer. Consequently, only frequencies and circumferential mode shapes could be calculated in our previous work, without changes along the axis of the silo, and no magnitudes of the vibrations. In addition, the turbulence modelling has been improved to resolve a fraction of the turbulent fluctuations. Finally, the results in our previous work overestimated the excitation by the flow in the wake of the silo group because the simulations were $2 \mathrm{D}$ which preserves large vortices and prevents their break up.

The goal of this paper is threefold. First, the necessity of taking into account the structural displacement in the flow calculations for this aeroelastic problem 
is analyzed. The differences between the results with and without including displacement in the flow will shed light on the mechanisms possibly causing the vibrations. Second, the effect of the group arrangement on the magnitude of the vibrations is determined by comparing by WSI simulations for a single silo with those for the entire closely spaced silo group. As mentioned above, the cooling towers at Ferrybridge experienced higher loading but they are more widely spaced so it is unclear whether that finding also applies to the silo group. Third, it is assessed whether the adopted modelling approach is suitable as an engineering tool for the analysis of new silo groups and possibly of similar structures to avoid the ovalling vibrations.

The paper is organized as follows. In section 2, details of the ovalling phenomenon are given and the present state of knowledge on wind-induced ovalling vibrations is briefly sketched. In section 3, the methodology for performing WSI simulations is explained in detail. The numerical models and techniques used for modelling both the wind flow and the silo structures are subsequently explained in section 4 . In section 5 , the results of the WSI simulations are described. Finally, the main conclusions of the study are given in section 6 .

\section{Observation and physical interpretation of ovalling vibrations}

Wind-induced ovalling vibrations have been observed on several empty silos at the windward side of a group consisting of 40 silos in the port of Antwerp (Belgium) during a storm on 27 October 2002. Based on observations and an in-depth analysis of available low resolution video footage of the ovalling event, the amplitudes of the ovalling shell oscillations were estimated in the order of $0.1 \mathrm{~m}$. Displacements of this order of magnitude are reasonably large compared to the silo diameter $D=5.5 \mathrm{~m}$ and the limited space of $0.3 \mathrm{~m}$ between adjacent silos. The largest vibrations were observed at the windward corner of the silo group (silo 1 in figure 2). Other silos at the windward side also showed ovalling but with smaller amplitude.

As shown in figure 3, the group of 8 by 5 thin-walled aluminium silos is 
mounted at $16.66 \mathrm{~m}$ above ground level. Only the upper cylindrical part of the silos is exposed to the incoming wind flow. The lower conical parts are embedded in a rectangular building that is $49 \mathrm{~m}$ long and $31.6 \mathrm{~m}$ wide. Each silo is $25 \mathrm{~m}$ high and is composed of aluminium sheets with a varying thickness decreasing with height from $10.5 \mathrm{~mm}$ to $6 \mathrm{~mm}$. Because the simulation of highly turbulent wind flows around complex geometries is a challenging task, simplifications in the computational model are required. Therefore pipes, cables, walking platforms on top of the silos as well as the staircase next to the group will not be considered in this analysis.

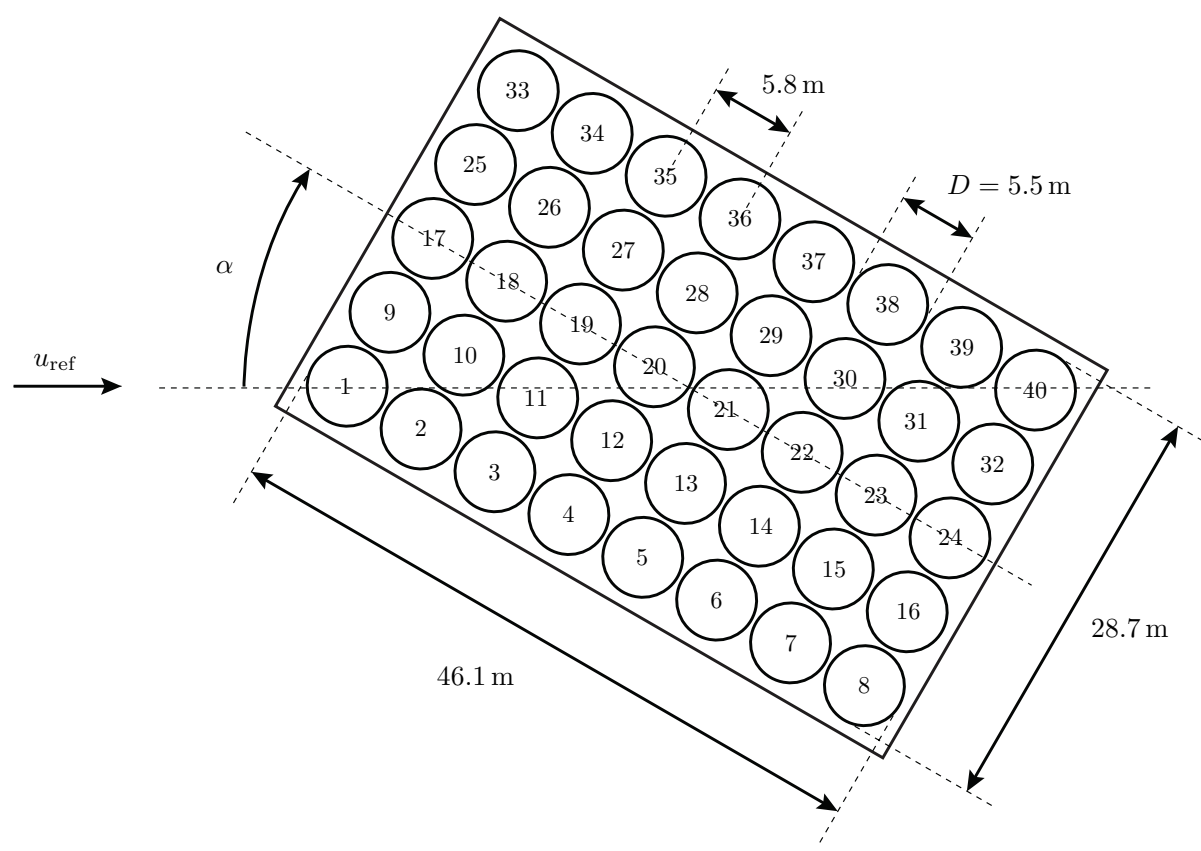

Figure 2: Plan view of the silo group with numbering of the individual silos. Normative dimensions are given as well as the definition of the angle of incidence $\alpha$.

No measurements are available of the wind field near the silo group at the time the ovalling instabilities were observed. However, at a permanent meteorological station in Deurne, about 7 kilometers east of the silo group, the hourly average wind speed was monitored and ranged from 61 to $68 \mathrm{~km} / \mathrm{h}$ with peaks up 


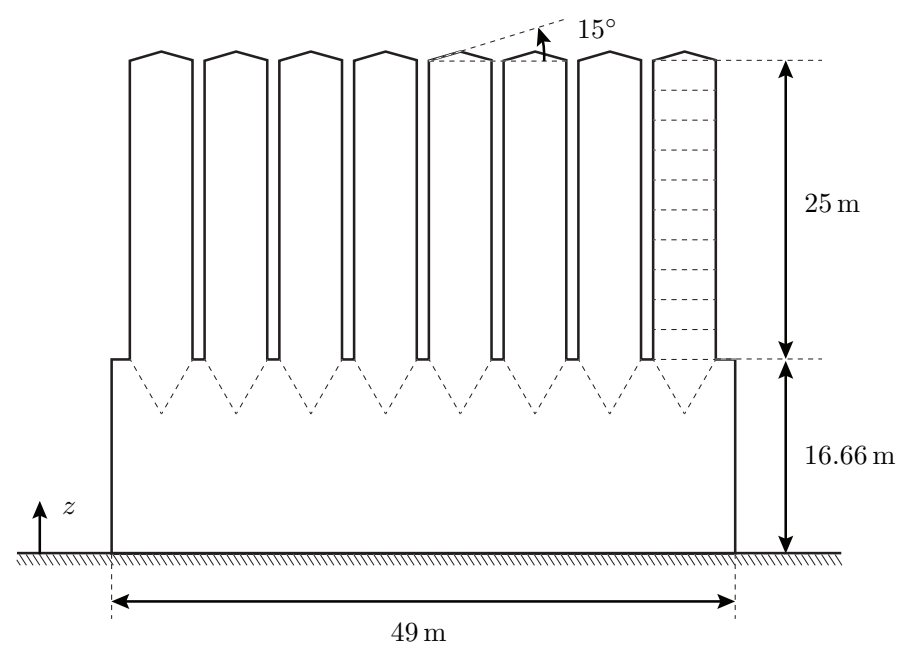

Figure 3: Lateral view of the silo group.

to $113 \mathrm{~km} / \mathrm{h}$. As indicated in figure 4, the silo group is located in flat surroundings near the river Scheldt and the main wind direction was west-southwest at the time of ovalling (figure 4 and $\alpha \approx 30^{\circ}$ in figure 2). With this information, a loading condition can be created which is expected to be similar to the one causing the observed ovalling in 2002 and sufficiently accurate to identify the underlying physical mechanisms that caused the vibrations.

A distinction is generally made between three different excitation mechanisms for flow-induced vibrations or more specifically wind-induced vibrations (Blevins, 1990; Païdoussis et al., 2011; Weaver et al., 1993; Žukauskas et al., 1988; Price et al., 1987): turbulence-induced vibrations, wake-induced vibrations and fluidelastic instability (FEI). In the case of wind, the latter is also called aeroelastic instability (AEI). Turbulence-induced excitation (or buffeting) originates from fluctuations in the wind flow around the structure, e.g. from natural turbulence in the wind flow attacking the structure. Wake-induced excitation is due to periodic vortex shedding in the wake of the structure, possibly with interaction (e.g. lock-in) between this wake and the structural motion. By contrast, aeroelastic phenomena are self-excited and the fluctuating forces are 


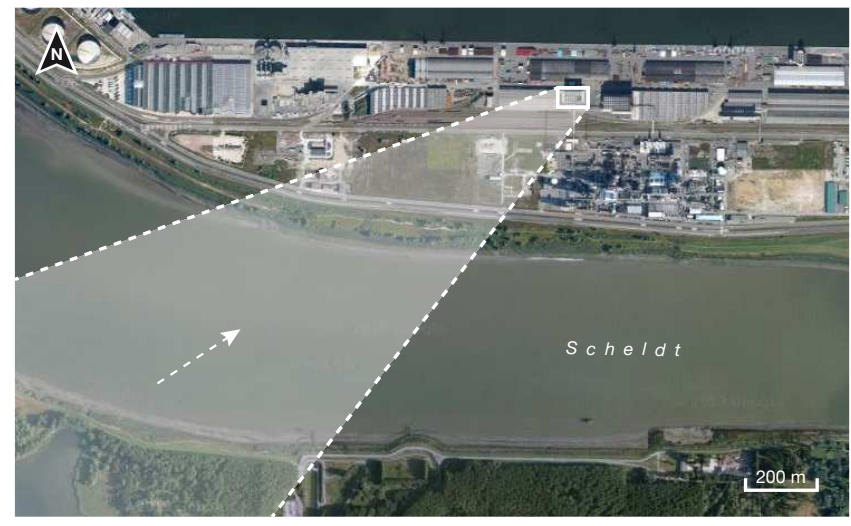

Figure 4: Location of the silo group near the river Scheldt in the port of Antwerp (Belgium). The mean wind direction during the storm of 27 October 2002 was west-southwest as indicated by the arrow. The shaded area denotes the flat region in front of the group.

134 due to structural motion. Also combinations of these phenomena occur.

\section{Methodology}

In this section, the one-way and two-way simulations are described. Comparing their results provides some insight into the excitation mechanisms of the observed wind-induced vibrations. The calculation of the silo's deformation by a structural solver and of the wind by a flow solver are discussed in more detail in section 4. In the following paragraph, short notations for these solvers are already defined.

The aerodynamic pressures and shear stresses $\mathbf{P}\left(t_{i}\right)$ on the interface between the structure and the wind flow - i.e. on the surface shared by the structural calculation and the wind flow calculation - are determined in a wind flow simulation for time step $t_{i}$. The structural solver $\mathcal{S}$ calculates the structural response of the silo due to these aerodynamic loads $\mathbf{P}\left(t_{i}\right)$ :

$$
\mathcal{S}\left[\mathbf{P}\left(t_{i}\right)\right]=\mathbf{U}\left(t_{i}\right)
$$

147 The response of the entire structure is calculated but only the displacements of the interface are passed back as $\mathbf{U}\left(t_{i}\right)$. The notation in this equation only 
mentions information on the interface ( $\mathbf{U}$ and $\mathbf{P}$ ). Obviously, also previous time levels $t_{i-1}, t_{i-2}, \ldots$ and additional boundary conditions are taken into account when calculating the solution, but these are not shown to keep the notation clear.

Based on the interface displacements, the flow solver $\mathcal{F}$ deforms the mesh in the fluid domain close to the silo. Subsequently, the flow solver calculates the wind in the entire fluid domain and returns the aerodynamic loading $\mathbf{P}\left(t_{i}\right)$ on the interface:

$$
\mathcal{F}\left[\mathbf{U}\left(t_{i}\right)\right]=\mathbf{P}\left(t_{i}\right)
$$

Similarly, the dependence on previous time levels, on additional boundary conditions and on the remainder of the fluid domain are implicitly understood.

\subsection{One-way approach}

In the one-way approach, the aerodynamic loads are first computed for a rigid structure, followed by calculation of the resulting structural response. Consequently, wind-structure interaction is not considered in the one-way approach. Because the structure is not moving in the aerodynamic load calculation, only turbulence-induced vibrations and part of the wake-induced vibrations are accounted for in this approach. Interactions between the wake-induced excitation and the structural motion as well as aeroelastic effects are not captured.

The flow solver $\mathcal{F}$ is used to determine the aerodynamic loading, $\mathbf{P}(t)$, acting on the interface between structure and wind flow in every time step: $\mathcal{F}[\mathbf{0}]=$ $\mathbf{P}\left(t_{i}\right)$, with $\mathbf{0}$ referring to the zero displacements of the rigid structure. As illustrated in figure 5 , the resulting time history $\mathbf{P}(t)$ of aerodynamic loads on the interface is subsequently applied as an external transient load on the structure and the structural displacements are computed in the structural solver: $\mathcal{S}[\mathbf{P}(t)]=\mathbf{U}(t)$.

Due to an abrupt application of wind pressures on the undeformed silo structures, a long period of transitional effects would be observed in the structural response. To avoid such a transitional regime, a static loading step is introduced first: $\mathbf{K} \mathbf{U}_{0}=\mathbf{P}_{0}$. The pressures in the first time step of the aerodynamic 


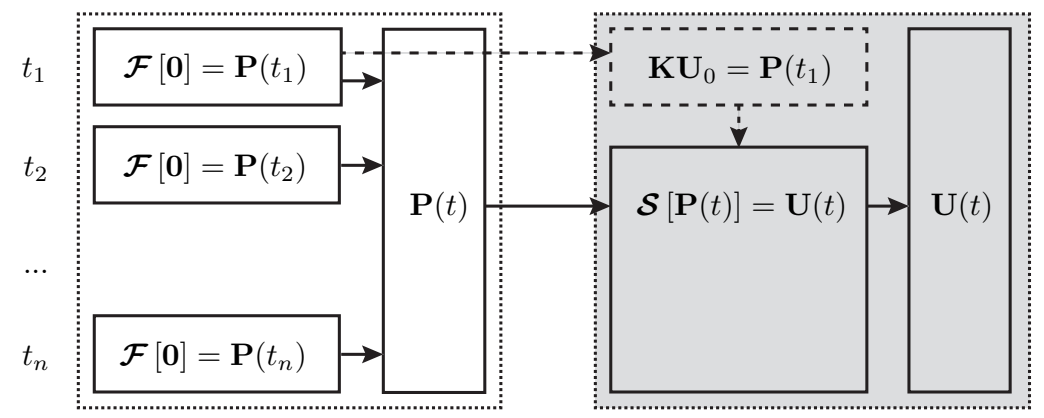

Figure 5: Schematic representation of the interaction between the flow solver $\mathcal{F}$ (white) and the structural solver $\mathcal{S}$ (grey) in the one-way partitioned simulation. The time-dependent aerodynamic loads $\mathbf{P}(t)$ are calculated with zero structural displacement. Subsequently, they are transferred to the structural solver for the calculation of the time-dependent displacement $\mathbf{U}(t)$, starting with initial displacement $\mathbf{U}_{0}$ from a steady calculation.

simulation $\mathbf{P}_{0}=\mathbf{P}\left(t_{1}\right)$ are statically applied to the structure and the structural response $\mathbf{U}_{0}$ is used as an initial condition for the dynamic structural calculations. The effect of such preliminary static calculation has been found to be very effective to reduce initial transitional effects in the present simulations.

\subsection{Two-way approach}

The two-way simulations take into account the structural motion when calculating the wind flow. As opposed to the one-way approach, these two-way simulations are therefore WSI (or FSI) simulations in the true sense. In addition to the turbulence-induced and part of the wake-induced vibrations, these simulations also capture aeroelastic effects and interactions between the wakeinduced excitation and the structural vibration. By comparing the results of the one-way and two-way simulations, it is possible to assess the importance of aeroelastic effects and/or interactions between the wake-induced excitation and the structural motion for the wind-induced vibrations.

In two-way simulations, the structural and flow solver are coupled in every time step as illustrated schematically in figure 6. A partitioned technique is applied which couples the black box flow solver with the black box structural 
solver. Partitioned means that two independent solvers are used for the flow equations and for the structural equations, as opposed to the monolithic approach which solves all equations together. Black box means that the source code of these solvers is not available and that Jacobians of the outputs with respect to the inputs cannot be obtained. Since the resulting aerodynamic loads $\mathbf{P}\left(t_{i}\right)$ and displacements $\mathbf{U}\left(t_{i}\right)$ typically differ slightly from the ones that were applied in the previous iteration within the same time step, consecutive flow and structural simulations have to be performed until equilibrium of loading and displacements is obtained on the interface between structure and wind flow.

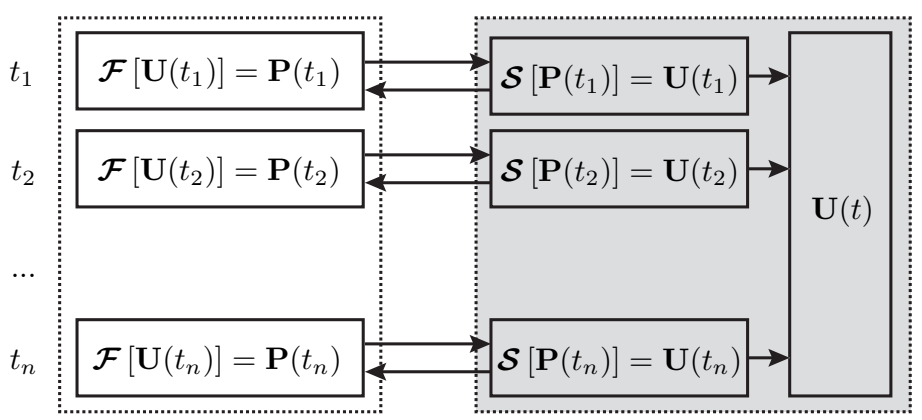

Figure 6: Schematic representation of the interaction between the flow solver $\mathcal{F}$ (white) and the structural solver $\mathcal{S}$ (grey) in the two-way partitioned approach. In each time step $t_{i}$, the displacements $\mathbf{U}\left(t_{i}\right)$ and loading $\mathbf{P}\left(t_{i}\right)$ on the silo surface are exchanged multiple times between the flow solver and the structural solver. When these coupling iterations have converged, the following time step is started.

A multitude of coupling techniques have been proposed to solve FSI problems in a partitioned way, as reviewed by Degroote (2013). In each time step, these techniques solve the flow equations and the structural equations multiple times to find the solution which satisfies the equilibrium conditions. For computational efficiency, the number of coupling iterations has to be limited as much as possible. Therefore, the interface quasi-Newton technique with an approximation for the inverse of the Jacobian of the coupled problem from a least-squares 
model (IQN-ILS) is applied (Degroote et al., 2009). This root-finding algorithm solves the equilibrium equation

$$
\mathcal{S}[\mathcal{F}[\mathbf{U}]]-\mathbf{U}=\mathbf{0}
$$

with Newton-Raphson iterations, using a least-squares approximation for the inverse of the Jacobian of this equation. Since wind flow and structural response are calculated in black box solvers, this approximation is exclusively based on displacements of the fluid-structure interface in previous coupling iterations and time steps.

\section{Numerical models of wind flow and structure}

As discussed in section 3, separate black box solvers can be used for the calculation of the wind flow and of the structural response. Details on the numerical methods and settings used by these solvers are discussed hereafter.

\subsection{Wind flow model}

In the 3D CFD model, two different configurations are considered: a single silo and a group of 40 silos, all of which are mounted on a prismatic building as described in section 2 . In the case of the single silo, the building is oriented at an angle of incidence of $\alpha=45^{\circ}$ with respect to the incoming wind flow while an angle of $\alpha=30^{\circ}$ is considered for the silo group configuration. The single silo case is studied in the light of comparing single silo and silo group results to assess the importance of the group effect on the ovalling vibrations. Furthermore, the single silo is a computationally less expensive problem that can be used to study grid sensitivity of the simulations.

Several best practice guidelines are available in the literature for the CFD simulation of wind flows in urban environments, e.g. the AIJ guidelines (AIJ, 2006; Tominaga et al., 2008) and the COST guidelines (COST Action C14, 2004; COST Action 732, 2007). Not only the size of the computational domain, but also the choice of appropriate boundary conditions, mesh and time step size as 
well as the choice of convergence criteria for iterative processes are discussed in these documents. These guidelines are followed as closely as possible.

Numerical procedure

A finite volume method is used in the CFD simulations for the discretization of the governing incompressible Navier-Stokes equations. Large eddy simulation (LES) resolves the large scale turbulent structures in the air flow. However, LES simulations are computationally demanding for high Reynolds number wind flows, especially if near-wall flows are calculated for a good prediction of the aerodynamic forces on the silo walls. By contrast, a Reynolds-averaged Navier Stokes (RANS) model is cheaper to simulate the near-wall flows. As a compromise between these two options, delayed detached eddy simulations (DDES) with $k-\omega$ SST as RANS model are performed in Ansys Fluent (Fluent 14.5, 2012). This turbulence model has also been used in other studies on similar objects and Reynolds numbers (Squires et al., 2008; Mannini et al., 2011b; Gopalan and Jaiman, 2015). (D)DES models are referred to as hybrid LES/RANS models because the RANS modelling of the boundary layer flow in the near-wall region is combined with the LES approach in the separated regions, where large unsteady turbulence scales are dominant. In the delayed DES approach, a shielding function is used to ensure that RANS is applied in the entire boundary layer since a sole geometrical separation of RANS and LES regions based on mesh size has shown to be insufficient (Spalart et al., 2006). For the shielding function, the blending functions of the SST turbulence model are used (Menter et al., 2003).

For the discretization of the momentum equations a bounded central differencing scheme is used. A second-order interpolation of the pressure, a secondorder upwind interpolation of the turbulent kinetic energy $k$ and the specific dissipation rate $\omega$ are applied. A bounded second-order implicit, unconditionally stable, time stepping method has been selected. The SIMPLE algorithm (Patankar and Spalding, 1972) is used for the pressure-velocity coupling between the momentum and the continuity equations. In the COST guidelines, it is rec- 
ommended that the residuals in the CFD solver should be reduced by at least four orders of magnitude. Therefore, the iterative process in (every coupling iteration within) every time step is truncated when the normalized residuals are all below $10^{-5}$.

In the one-way simulations, an Eulerian, fixed grid description is used in the fluid solver. Modifications have to be made to account for a moving fluid grid when a two-way WSI simulation is carried out. Therefore, the flow equations are reformulated in an arbitrary Lagrangian-Eulerian (ALE) description (Donea et al., 1982, 2004). The mesh motion in the wind domain is determined by the motion of the deformable fluid-structure interface. For the simulations with a single silo, a smoothing technique (Batina, 1990; Löhner and Yang, 1996) is used to extend the displacements of the fluid-structure interface into the entire fluid domain. Conversely, for the silo group, Laplace equations are solved for the mesh displacement to handle the more challenging ALE mesh updates in the narrow gaps between adjacent silos. This implies that these diffusion equations for the mesh velocities of the fluid grid have to be solved iteratively in every coupling iteration. Approximately 10 iterations are required for every Laplace mesh update in the present application. This results in good grid quality for all occurring displacements of the silos and causes only a small increase in simulation time compared to the Eulerian formulation.

\section{Computational domain and boundary conditions}

The size of the computational domain is shown in figure 7 and is slightly larger than prescribed by guidelines (AIJ, 2006): e.g. height of the domain $6 H>5 H$, blockage ratio $1.7 \%<3 \%$, etc. The dimensions of the domain do not only depend on the zone of interest but also on the applied boundary conditions. The top boundary condition has to sustain the equilibrium ABL profiles. For this purpose, symmetry boundary conditions are applied which provide a good approximation of reality as long as the top boundary is located sufficiently far from the region of interest (COST Action 732, 2007). Symmetry boundary conditions are applied at the lateral boundaries as well while a constant pressure 


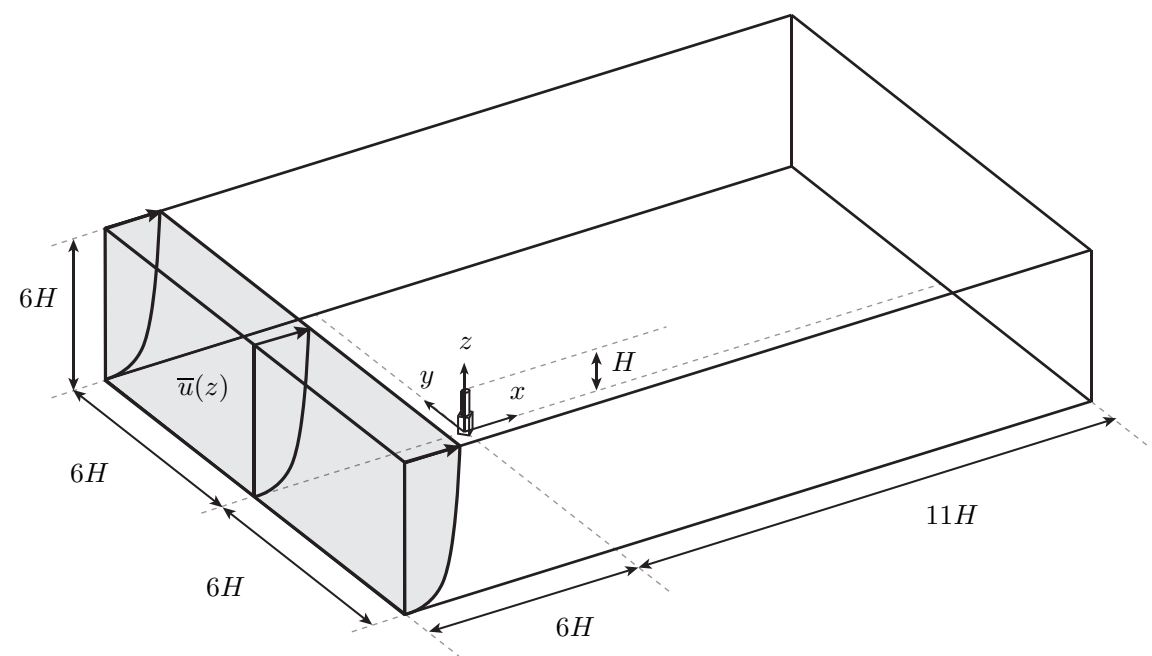

Figure 7: Dimensions of the computational domain and global coordinate system, with origin at the bottom of the domain at the centre of the structure.

is applied at the outflow boundary. The walls of the building and the silo structures are modelled as no-slip walls.

A horizontally homogeneous unidirectional wind flow is applied at the inlet of the domain. Since no exact wind field data are available, approximative wind conditions are set up based on guidelines (AIJ, 2006; BIN, 2010; Tominaga et al., 2008; ANSI, 2010). Given the location of the silo group, the present case is classified in terrain category II of Eurocode 1 (BIN, 2010). The free field wind velocity is set to $u_{\mathrm{ref}}=31.8 \mathrm{~m} / \mathrm{s}$ at approximately mid-height of the silos, i.e. at $z_{\text {ref }} \approx 30 \mathrm{~m}$ above ground level. This corresponds roughly to the peak wind velocities that were monitored in 2002 at the meteorological station in Deurne a few kilometers from the site. The highly turbulent wind flow regime around the group of silos is hence post-critical (Zdravkovich, 1997) at Reynolds number $\operatorname{Re}=u_{\text {ref }} D / \nu=1.24 \times 10^{7}$ with $\nu=1.41 \times 10^{-5} \mathrm{~m}^{2} / \mathrm{s}$.

For the variation of the mean wind velocity with height $\bar{u}(z)$ in the atmospheric boundary layer (ABL, cfr. shaded area in figure 7), a power law profile as prescribed in the AIJ guidelines is used (AIJ, 2006; Tominaga et al., 2008). 
The mean wind velocity $u_{\text {ref }}$ at a reference height of $z_{\text {ref }}=30 \mathrm{~m}$ is used to fit the power law:

$$
\bar{u}(z)=u_{\text {ref }}\left(\frac{z}{z_{\text {ref }}}\right)^{\beta},
$$

with $\beta$ a constant depending on the terrain roughness which equals 0.14 for the considered terrain category (II, open country).

Note that an incompatibility of inflow data and wall boundary conditions at ground level may have an important impact on the preservation of the homogeneity of the ABL flow (Blocken and Carmeliet, 2006; COST Action C14, 2004; COST Action 732, 2007; Quinn et al., 2001; Zhang, 1994). This effect is limited to the lowest region of the ABL however (Castro, 2003). In the German guidelines (VDI-Richtlinie 3783, 2003), it is therefore recommended to place at least two nodes between the ground and the zone of interest. Considering the fact that the silo structures are mounted on a prismatic building, this effect is of no further consequence in the present simulations.

To simulate a 3D turbulent wind flow, realistic random wind field data have to be modelled at the inlet of the domain as well. In the present simulations, turbulence is synthetically generated with a spectral synthesizer method proposed by Smirnov et al. (2001), as implemented in Ansys Fluent (Fluent 14.5, 2012). A power law profile for the turbulence intensity $I_{u}(z)$, based on the AIJ guidelines (Tominaga et al., 2008) is used as input for this spectral synthesizer method:

$$
I_{u}(z)=0.1\left(\frac{z}{z_{\mathrm{G}}}\right)^{-\beta-0.05},
$$

with $z_{\mathrm{G}}=350 \mathrm{~m}$ the gradient height of the ABL in open country (terrain category II) (Tominaga et al., 2008).

\section{Discretization sensitivity analysis}

In the case of DDES simulations, it is advisable to get an indication of the sensitivity of the results to discretization cut-offs. In tables 1 and 2 , the size of the computational domain is listed for several levels of grid refinement in the simulation of the flow around the single silo and around the entire silo group, 
respectively. Most of the grid is block-structured, except for the part at the top of the silos. The maximal $y^{+}$values along the circumference are around 100, so wall functions are applied. At least 60 cells are present in the gaps between the silos of the group. It is obvious that an increase in number of grid cells results in a significant increase of computational efforts. It takes about 40, 72 and 111 hours of computing time for the coarse, medium and fine meshes, respectively, to calculate $40 \mathrm{~s}$ of wind flow in the 3D simulation for a single silo.

These calculations have been performed on 64 parallel cores, i.e. half of the cores of four quad-socket octa-core AMD Magny-Cours machines (Opteron 6136) at $2.4 \mathrm{GHz}$ with almost full use of the working memory of all machines $(64 \mathrm{~GB}$ RAM per machine). For the simulation of the wind flow around the entire silo group on the coarse and fine grids, respectively 280 and 450 hours are required to simulate the same time frame of $40 \mathrm{~s}$ on the same 64 parallel cores.

\begin{tabular}{lcc}
\hline Mesh & $N_{\text {circ }}$ & $N_{\text {dom }}$ \\
\hline coarse & 64 & 1573872 \\
medium & 80 & 4552486 \\
fine & 112 & 8233532 \\
\hline
\end{tabular}

Table 1: Overview of the number of cells $N_{\text {dom }}$ in the computational domain for the single silo simulations. Three stages of grid refinement are considered, based on the number of divisions $N_{\text {circ }}$ on the circumference of the silo surface.

\begin{tabular}{lcc}
\hline Mesh & $N_{\text {circ }}$ & $N_{\text {dom }}$ \\
\hline coarse & 64 & 8276472 \\
fine & 80 & 17066268 \\
\hline
\end{tabular}

Table 2: Overview of the number of cells $N_{\text {dom }}$ in the computational domain for the silo group simulations. Two stages of grid refinement are considered, based on the number of divisions $N_{\text {circ }}$ on the circumference of the silo surface.

In view of the objectives of the present investigation, this inevitably implies 


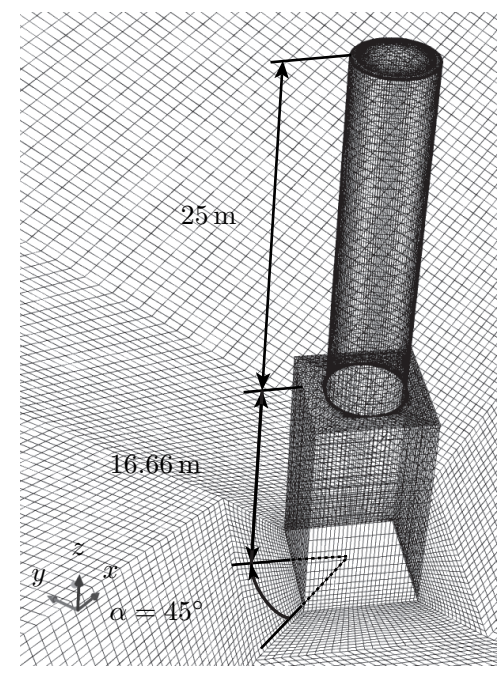

Figure 8: Detail of the coarse mesh of a single silo structure with the wind at an angle of incidence $\alpha=45^{\circ}$.

that compromises between accuracy and required computational effort have to be made, especially for the two-way simulations where multiple coupling iterations have to be performed per time step. In this respect, it is important to assess discretization errors and take them into account for the interpretation of simulation results rather than pursuing extreme grid refinements to obtain a completely grid independent solution. Furthermore, it is noted that a simulated time frame of only $40 \mathrm{~s}$ is fairly short in wind engineering where typically response quantities are averaged over periods of 10 minutes or longer. Again, the length of these time intervals is limited by the high computational costs.

First, the single silo configuration is considered. A detail of the computational mesh (coarse grid) for the single silo is shown in figure 8 , with incoming flow along the $x$-axis. The results for the mean and RMS values of the drag coefficients in $x$ - and $y$-directions are summarized in table 3. A variability in the drag coefficients for the different grid sizes is observed. These discrepancies are due to differences in the predicted location of the separation point on the silo surface and, hence, the largest differences are observed for the mean drag 
coefficient $\bar{C}_{\mathrm{d} x}$ in the $x$-direction. It should be underlined that the accurate prediction of separation points on smooth, curved surfaces is a major challenge for every turbulence modelling technique in CFD.

\begin{tabular}{lcccc}
\hline Mesh & $\bar{C}_{\mathrm{d} x}$ & $C_{\mathrm{d} x}^{\mathrm{RMS}}$ & $\bar{C}_{\mathrm{d} y}$ & $C_{\mathrm{d} y}^{\mathrm{RMS}}$ \\
\hline Coarse & 0.426 & 0.021 & 0.025 & 0.061 \\
Medium & 0.359 & 0.018 & 0.018 & 0.052 \\
Fine & 0.302 & 0.017 & -0.005 & 0.027 \\
\hline
\end{tabular}

Table 3: Mean value and root mean square value of the drag coefficients $C_{\mathrm{d} x}$ and $C_{\mathrm{d} y}$ for the single silo with wind flow at an angle of incidence $\alpha=45^{\circ}$ and for different mesh refinements.

For a selection of representative silos in the group, the drag coefficients in $x$ - and $y$-directions are listed in table 4. Figure 9 furthermore shows the time history of $C_{\mathrm{d} x}$ and $C_{\mathrm{d} y}$ for the corner silos of the group. From table 4 , it is observed that the cylinders at the front of the silo group (e.g. silos 1, 17, 25 and 33) are subject to larger drag forces than silos at the centre of the group (e.g. silos 30 and 38). Silos 1, 9, 17, 25 and 33 have the largest area exposed directly to the incoming flow (figure 2), resulting in the highest drag coeffients $C_{\mathrm{d} x}$. Overall, it is concluded that the drag coefficients predicted on both meshes are in reasonably good agreement for the majority of the silos, including for silo 1 where the ovalling vibrations have been observed. Although the separation of the flow is much more geometrically determined for the entire silo group, difficulties with the prediction of separation are still observed for the transverse corner silos (silos 8 and 33 in figure 2) and for some of the upwind silos in the first row of the group. This difference between the results on both meshes at the transverse corner silos is mainly due to the prediction of the separation points on smooth, curved surfaces as in the single silo case.

Finally, also the influence of the time step on the accuracy of the simulations has been assessed by performing different simulations with a systematic reduction of the time step. This time step refinement has only been carried out for the single silo configuration. From the results summarized in table 5, 

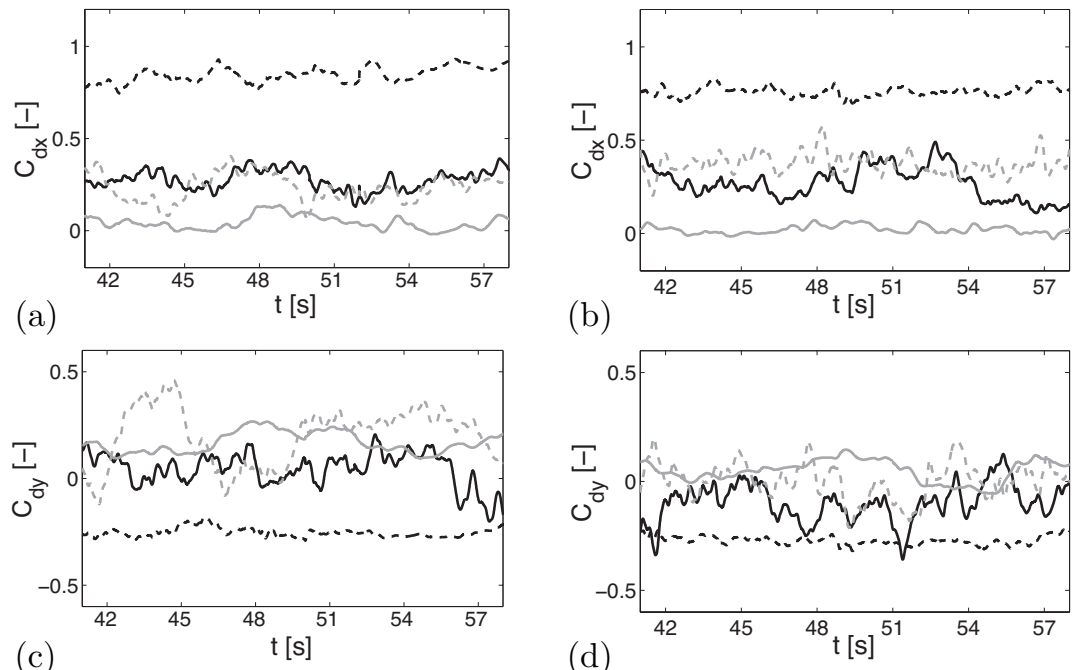

Figure 9: Time history of the drag coefficients (top) $C_{\mathrm{d} x}$ and (bottom) $C_{\mathrm{d} y}$ for the corner silos of the silo group (silo 1 - dashed black line; silo 8 - solid black line; silo 33 - dashed grey line; silo 40 - solid grey line) with wind flow at an angle of incidence $\alpha=30^{\circ}$ and for (left) the coarse mesh and (right) the fine mesh.

it is concluded that a time step $\Delta t=0.005 \mathrm{~s}$ is sufficiently small. Although no explicit verification was made for the entire silo group, it can be reasoned that all physical flow phenomena for the silo group have larger length scales and hence longer periods which can be accurately captured by this time step. The COST guidelines (COST Action C14, 2004; COST Action 732, 2007) instruct that the choice of the time step is determined by the relevant frequencies in the flow: the highest frequency to be considered needs to be resolved with at least 10 to 20 time steps. When a time step size of $0.005 \mathrm{~s}$ is used, this means that frequencies of $10 \mathrm{~Hz}$ are still resolved. In the following, it will be shown that this is sufficiently accurate given the low natural frequencies of the silo structures and the low frequency content of the wind flow.

\subsection{Structural model}

The software package Abaqus (Abaqus 6.10, 2010) has been used to construct a finite element (FE) model of the silo structure which allows to calculate the 


\begin{tabular}{|c|c|c|c|c|c|}
\hline Silo & Mesh & $\bar{C}_{\mathrm{d} x}$ & $C_{\mathrm{d} x}^{\mathrm{RMS}}$ & $\bar{C}_{\mathrm{d} y}$ & $C_{\mathrm{d} y}^{\mathrm{RMS}}$ \\
\hline \multirow{2}{*}{1} & Coarse & 0.841 & 0.045 & -0.253 & 0.022 \\
\hline & Fine & 0.781 & 0.043 & -0.268 & 0.022 \\
\hline \multirow{2}{*}{5} & Coarse & 0.221 & 0.048 & 0.199 & 0.045 \\
\hline & Fine & 0.233 & 0.057 & 0.192 & 0.043 \\
\hline \multirow{2}{*}{8} & Coarse & 0.409 & 0.121 & -0.094 & 0.154 \\
\hline & Fine & 0.361 & 0.128 & -0.269 & 0.195 \\
\hline \multirow{2}{*}{17} & Coarse & 0.757 & 0.053 & -0.381 & 0.031 \\
\hline & Fine & 0.798 & 0.057 & -0.411 & 0.045 \\
\hline \multirow{2}{*}{25} & Coarse & 0.704 & 0.055 & -0.269 & 0.043 \\
\hline & Fine & 0.748 & 0.061 & -0.281 & 0.050 \\
\hline \multirow{2}{*}{30} & Coarse & 0.036 & 0.030 & 0.037 & 0.017 \\
\hline & Fine & 0.038 & 0.031 & 0.043 & 0.024 \\
\hline \multirow{2}{*}{33} & Coarse & 0.254 & 0.114 & 0.175 & 0.166 \\
\hline & Fine & 0.403 & 0.072 & 0.059 & 0.096 \\
\hline \multirow{2}{*}{38} & Coarse & 0.069 & 0.035 & 0.066 & 0.028 \\
\hline & Fine & 0.043 & 0.034 & 0.064 & 0.033 \\
\hline \multirow{2}{*}{40} & Coarse & 0.050 & 0.046 & 0.152 & 0.061 \\
\hline & Fine & 0.042 & 0.048 & 0.104 & 0.066 \\
\hline
\end{tabular}

Table 4: Mean value and root mean square value of the drag coefficients $C_{\mathrm{d} x}$ and $C_{\mathrm{d} y}$ for a selection of silos of the 8 by 5 silo group with wind flow at an angle of incidence $\alpha=30^{\circ}$ and for the coarse and the fine mesh. The silos on the corners of the group are highlighted in the table.

408

structural response of the silos to applied aerodynamic pressures and shear stresses. This model is also used to determine the ovalling eigenmodes and corresponding natural frequencies of the silos.

Each silo in the group is made of 10 aluminium cylindrical sheets with a height of $2.5 \mathrm{~m}$ each (figure 10). At the top and bottom of the cylinder, a cone is welded to the cylinder at an angle of $15^{\circ}$ and $60^{\circ}$ with the horizontal plane, re- 


\begin{tabular}{lcc}
\hline$\Delta t$ & $C_{\mathrm{d} x}^{\mathrm{RMS}}$ & $\Delta C_{\mathrm{d} y}$ \\
\hline $0.01 \mathrm{~s}$ & 0.022 & 0.104 \\
$0.005 \mathrm{~s}$ & 0.016 & 0.085 \\
$0.0025 \mathrm{~s}$ & 0.016 & 0.082 \\
$0.000125 \mathrm{~s}$ & 0.016 & 0.082 \\
\hline
\end{tabular}

Table 5: Root mean square value of drag coefficient $C_{\mathrm{d} x}$ and amplitude $\Delta C_{\mathrm{d} y}=C_{\mathrm{d} y}^{\max }-C_{\mathrm{d} y}^{\min }$ for the 3D single silo (fine mesh) and for different time steps.

spectively. Shell elements with linear FE interpolation functions are used for the entire structure. For the dynamic simulations, the unconditionally stable and second-order accurate Hilbert-Hughes-Taylor (HHT) method as implemented in the Abaqus FE solver is used.

The following material properties are used for aluminium: density $\rho=$ $2700 \mathrm{~kg} / \mathrm{m}^{3}$, Young's modulus $E=67.6 \mathrm{GPa}$ and Poisson's ratio $\nu=0.35$. Rayleigh damping is assumed and based on a constant damping ratio $\xi=0.75 \%$ for the two lowest eigenmodes. This is a realistic approximation since modal damping ratios $\xi$ for this structure, determined during in situ measurements by Dooms et al. (2006), were found to vary between $0.07 \%$ and $1.32 \%$. These low modal damping values are typical for a welded aluminium structure. The silo is bolted to an octogonal steel framework at 4 points on the circumference of the cylindrical shell (figure 10).

To accommodate an easy transfer of the aerodynamic loads on the silo walls from flow solver to structural solver, the mesh of the FE model is chosen conforming to the mesh on the silo walls in the CFD simulations. As a result, three different structural models are used, i.e. one for each of the three CFD grids for a single silo (cfr. table 1).

Natural frequencies and ovalling modes

The mass normalized eigenmodes $\boldsymbol{\Phi}$ and corresponding eigenfrequencies $f_{\text {eig }}$ are determined by solving the generalized eigenvalue problem of the structure. 


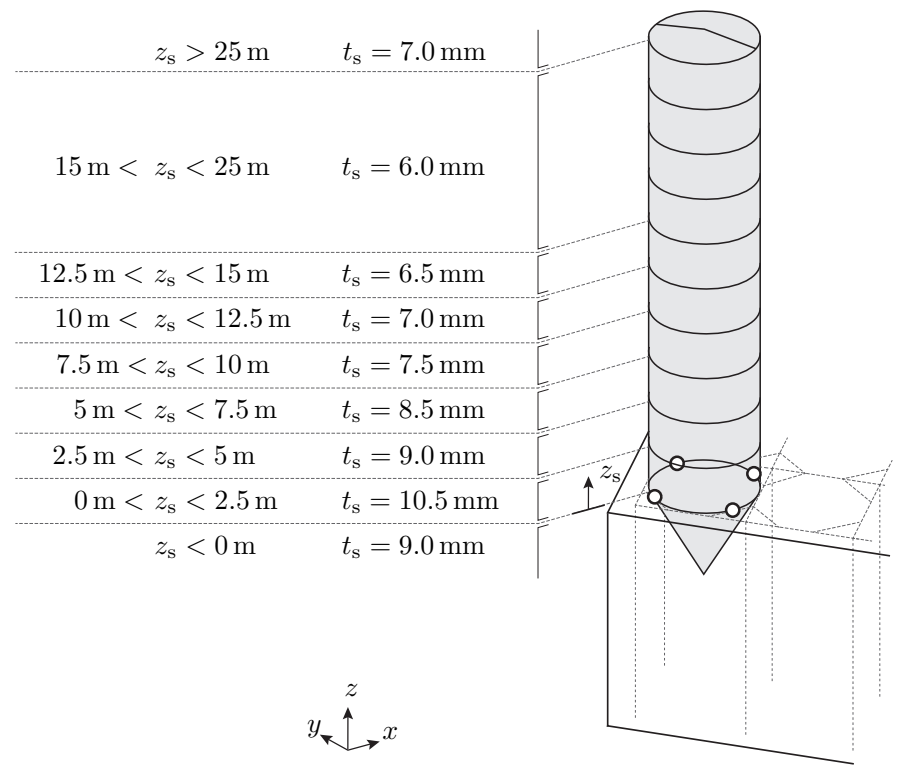

Figure 10: Geometrical respresentation of the silo structure with indication of the variation of the shell thickness $t_{\mathrm{s}}$ of the aluminium plates along the height $z_{\mathrm{s}}$ of the silo structure.

The eigenmodes corresponding to the lowest eigenfrequencies are summarized in table 6 and a selection of these modes is shown in figure 11.

\begin{tabular}{ccc|ccc|ccc}
\hline $\boldsymbol{\Phi}_{j}$ & $(m, n)$ & $f_{\text {eig } j}[\mathrm{~Hz}]$ & $\boldsymbol{\Phi}_{j}$ & $(m, n)$ & $f_{\text {eig } j}[\mathrm{~Hz}]$ & $\boldsymbol{\Phi}_{j}$ & $(m, n)$ & $f_{\text {eig } j}[\mathrm{~Hz}]$ \\
\hline $\boldsymbol{\Phi}_{1}$ & $(1,3)$ & 3.96 & $\boldsymbol{\Phi}_{7}$ & $(1,5)$ & 5.70 & $\boldsymbol{\Phi}_{13}$ & $(2,5)$ & 8.19 \\
$\boldsymbol{\Phi}_{2}$ & $(1,3)$ & 3.97 & $\boldsymbol{\Phi}_{8}$ & $(1,5)$ & 5.71 & $\boldsymbol{\Phi}_{14}$ & $(2,6)^{*}$ & 8.62 \\
$\boldsymbol{\Phi}_{3}$ & $(1,4)$ & 3.99 & $\boldsymbol{\Phi}_{9}$ & $(1,6)$ & 7.72 & $\boldsymbol{\Phi}_{15}$ & $(2,4)$ & 8.85 \\
$\boldsymbol{\Phi}_{4}$ & $(1,4)$ & 4.11 & $\boldsymbol{\Phi}_{10}$ & $(1,6)$ & 7.72 & $\boldsymbol{\Phi}_{16}$ & $(2,4)$ & 9.10 \\
$\boldsymbol{\Phi}_{5}$ & $(1,5)$ & 5.34 & $\boldsymbol{\Phi}_{11}$ & $(1,2)$ & 7.83 & $\boldsymbol{\Phi}_{17}$ & $(2,6)$ & 9.62 \\
$\boldsymbol{\Phi}_{6}$ & $(1,5)$ & 5.35 & $\boldsymbol{\Phi}_{12}$ & $(2,5)$ & 8.18 & $\boldsymbol{\Phi}_{18}$ & $(2,6)$ & 9.72 \\
\hline
\end{tabular}

Table 6: Natural frequencies $f_{\text {eig }}$ of the lowest ovalling eigenmodes of the silo structure. The mode shapes indicated with an asterisk are a combination of two 'pure' ovalling shapes.

The mode shapes are of the ovalling type and are referred to by a couple $(m, n)$, where $m$ denotes the number of half wavelengths in the axial direction 
and $n$ is the number of circumferential wavelengths. For an axisymmetric structure most of the ovalling eigenmodes come in pairs: e.g. $\boldsymbol{\Phi}_{1}$ and $\boldsymbol{\Phi}_{2}$ are both classified as mode shapes $(1,3)$ but are mutually orthogonal. The mode shapes indicated with an asterisk, e.g. $\boldsymbol{\Phi}_{14}=(2,6)^{*}$ are referred to as 'hybrid' mode shapes. Such mode shapes are characterized by two combined 'pure' ovalling shapes at once, e.g. shapes $(1,2)$ and $(2,6)$ in the case of $\boldsymbol{\Phi}_{14}=(2,6)^{*}$ (figure 11d).

From the silo group vibrations observed during the storm in 2002, it is believed that mainly ovalling modes $(1,3)$ and $(1,4)$ were excited by the wind field, corresponding to the lowest eigenfrequencies of the silo structure. Measurements during normal wind loading have also shown that eigenmodes with 3 or 4 circumferential wavelengths have the highest contribution to the response of the silos (Dooms et al., 2006).

For the physical interpretation of the structural response, a more quantitative analysis is proposed in the following that allows to assess the modal contributions in the response. The deformation energy $E_{\mathrm{d}}(t)$ is easily determined from the calculated structural response $\mathbf{U}(t)$ of the entire structure:

$$
E_{\mathrm{d}}(t)=\frac{1}{2} \mathbf{U}^{\mathrm{T}}(t) \mathbf{K} \mathbf{U}(t)
$$

where $\mathbf{K}$ represents the stiffness matrix of the structural FE model. The contributions of the different eigenmodes in the response are subsequently distinguished by applying modal decomposition. By inserting $\mathbf{U}(t)=\boldsymbol{\Phi} \boldsymbol{\alpha}(t)$, with $\boldsymbol{\alpha}(t)$ the modal coordinates, in equation (6), the deformation energy is decomposed into modal contributions as follows:

$$
E_{\mathrm{d}}(t)=\frac{1}{2} \boldsymbol{\alpha}^{\mathrm{T}}(t) \boldsymbol{\Phi}^{\mathrm{T}} \mathbf{K} \boldsymbol{\Phi} \boldsymbol{\alpha}(t)=\frac{1}{2} \sum_{j=1}^{n_{\mathrm{DOF}}} \omega_{j}^{2} \alpha_{j}^{2}(t)=\sum_{j=1}^{n_{\mathrm{DOF}}} E_{\mathrm{d} j}(t)
$$

where $n_{\mathrm{DOF}}$ is the number of degrees of freedom of the FE model. Based on this expression, the contribution $E_{\mathrm{d} j}(t)$ of an individual mode $j$ to the deformation energy $E_{\mathrm{d}}(t)$ can be determined from the modal coordinates $\boldsymbol{\alpha}(t)$. 

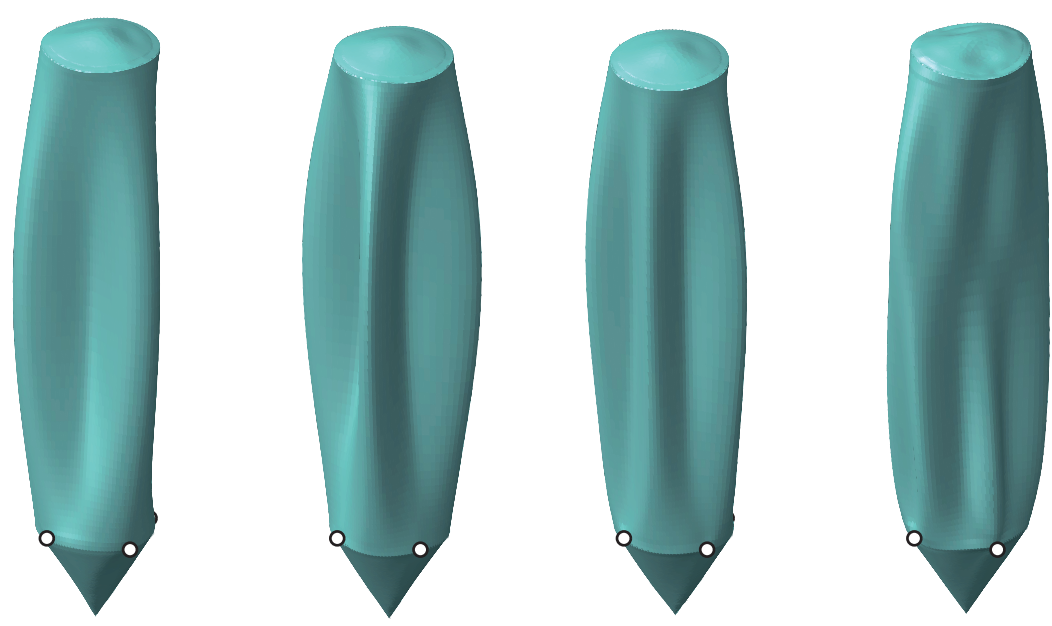

(a)

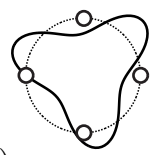

(b)

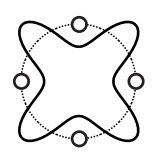

(c)

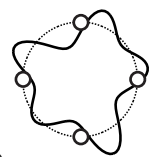

(d)

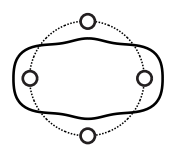$$
\text { (d) }
$$

Figure 11: (top) 3D isotropic view and (bottom) horizontal section at mid-height for a selection of ovalling eigenmodes of a silo: (a) mode $\boldsymbol{\Phi}_{1}=(1,3)$ at $3.96 \mathrm{~Hz}$, (b) mode $\boldsymbol{\Phi}_{4}=(1,4)$ at $4.11 \mathrm{~Hz}$, (c) mode $\boldsymbol{\Phi}_{5}=(1,5)$ at $5.34 \mathrm{~Hz}$ and $(\mathrm{d}) \operatorname{mode} \boldsymbol{\Phi}_{14}=(2,6)^{*}$ at $8.62 \mathrm{~Hz}$.

\section{Wind-structure interaction simulation results}

In this section, the wind-induced vibrations of the silos are analyzed using wind-structure interaction simulations. As described in section 3 both one-way and two-way simulations are performed for the single silo and the silo group configuration. Given the agreement between the results on the different grids in the previous section, the second finest grid is presented here. Simulations performed on other grids led to the same conclusions and are hence omitted for brevity. A time frame with the last $25 \mathrm{~s}$ is considered in all coupled simulations unless indicated differently. 


\subsection{Single silo simulations}

\section{One-way simulations}

First, the flow pattern around the single silo as shown in figure 12 is considered. Streamlines are shown in a vertical and several horizontal planes across the height of the structure. The flow has a highly three-dimensional character and different flow patterns can be observed at different heights. Upstream of the structure, near the ground, a horseshoe vortex is clearly formed in the flow (figures 12top and a). The flow around the prismatic building is attached to the surface on the windward side of the prism and separation occurs at the corners of the building. At the connection between the prismatic building and the cylindrical silo structure, the flow is deflected upwards and detaches from the sharp edges of the building. These accelerated upward flows are deflected sideways due to the vicinity of the cylindrical silo (figure 12b). Along the height of the silo structure, the flow is separated at the lee side of the cylindrical surface, generating a highly turbulent and narrow wake region, typical for high Reynolds number cross-flows around cylinders. At the free end of the silo structure (figure 12d), the separated trailing vortex is mainly dragged downstream and only slightly deflected downwards. The existing downwash effect has already disappeared at mid-height of the silo (figure 12c). As indicated by Park and Lee (2004), the conical shape of the silo top might also explain the reduced width of the wake formed near the free end of the cylinder. Downwash effects are therefore thought to be limited at the present Reynolds number and little if any interaction with the flow at midspan of the silo is observed. It could also be argued that downwash effects can only have a very limited effect on the already narrow and short wake region over the entire length of the silo structure.

In the one-way simulation of the single silo, a predominantly static structural response with maximal displacements of approximately $0.04 \mathrm{~m}$ is found and significantly smaller ovalling vibrations in the order of $0.01 \mathrm{~m}$ are observed. These deformations are converted into nodal contributions to the deformation energy $E_{\mathrm{d} j}(t)$ which are shown as a function of time in figure 13 for the 20 
(d)

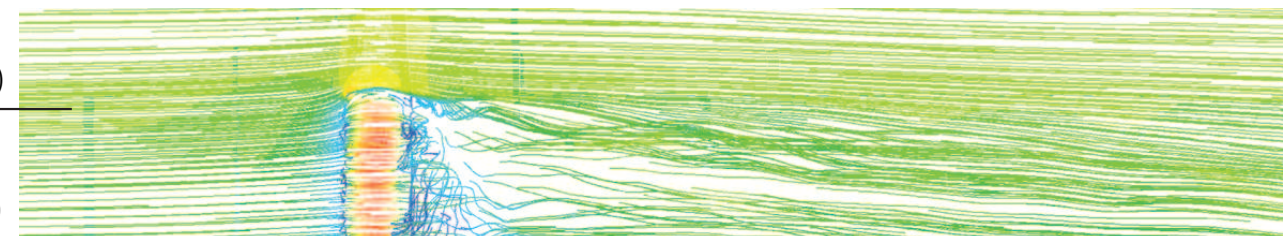

(c)

(b)

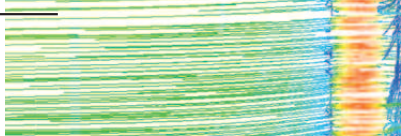

(a)

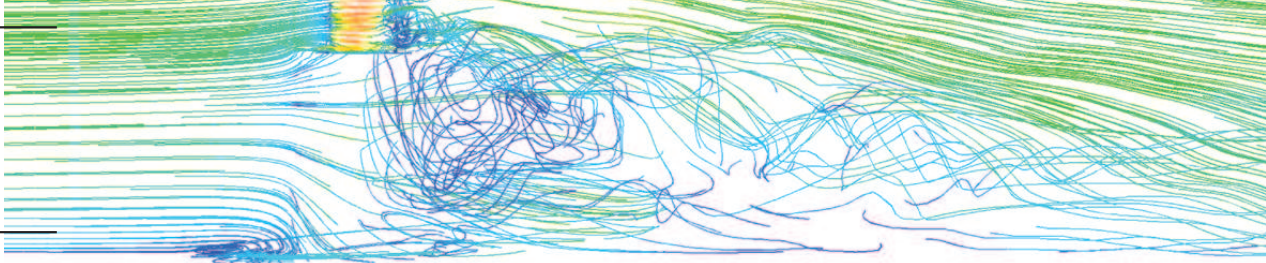

(a)
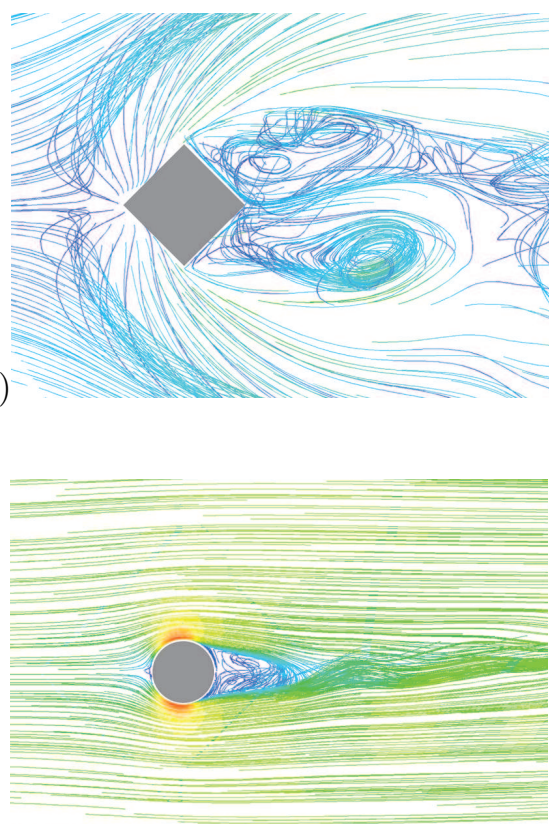

(c)

Figure 12: Streamlines of the wind flow around a single silo structure after $40 \mathrm{~s}$, colored according to the velocity magnitude, released from (top) a vertical plane $y=0 \mathrm{~m}$ and horizontal planes at (a) $z=0.5 \mathrm{~m}$, (b) $z=17.16 \mathrm{~m}$, (c) $z=29.16 \mathrm{~m}$, and (d) $z=41.16 \mathrm{~m}$. 
structural eigenmodes with the lowest eigenfrequencies. Additional calculations accounting for geometrical nonlinearities did not reveal a significant effect of nonlinearities on the structural response.

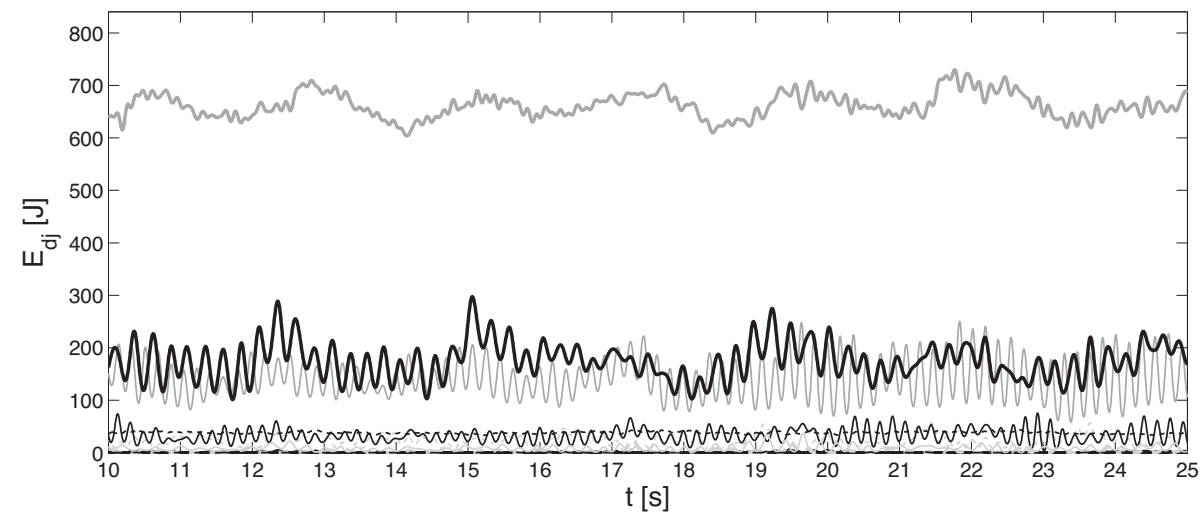

Figure 13: Modal deformation energy $E_{\mathrm{d} j}(t)$ of the first 20 mode shapes, computed from the structural response in the one-way simulation of a single silo: $\boldsymbol{\Phi}_{2}=(1,3)$ (bold black line), $\boldsymbol{\Phi}_{4}=(1,4)$ (thin black line), $\boldsymbol{\Phi}_{6}=(1,5)$ (thin grey line), $\boldsymbol{\Phi}_{14}=(2,6)^{*}$ (bold grey line) $\boldsymbol{\Phi}_{18}=(2,6)$ (dashed black line), and the remaining mode shapes $\boldsymbol{\Phi}_{j}$ (dashed grey lines, with small energy content).

Figure 13 shows that only a limited number of modes contribute significantly to the structural response of the silo. Typically, three time scales, named mean, resonant and quasi-static, are distinguished in the modal deformation energy $E_{\mathrm{d} j}(t)($ Holmes, 2015).

- The mean, time averaged modal deformation energy is related to the static excitation of a structural mode. Mode $\boldsymbol{\Phi}_{18}=(2,6)$, for example, is almost exclusively excited statically (dashed black line in figure 13).

- While a whole range of irregular oscillations seems to be present in the fluctuating parts of the response, regular oscillations of eigenmodes $\boldsymbol{\Phi}_{2}=$ $(1,3)$ (bold black line), $\boldsymbol{\Phi}_{4}=(1,4)$ (thin black line) and $\boldsymbol{\Phi}_{6}=(1,5)$ (thin grey line) at their respective eigenfrequencies can be clearly observed. This resonant response is mainly found for the eigenmodes with the lowest 
natural frequencies.

- All remaining oscillations are categorized as the background response of the structure. The fourteenth mode $\boldsymbol{\Phi}_{14}=(2,6)^{*}$, for example, is almost exclusively excited by low frequency fluctuations (bold grey line in figure 13). Because this is a one-way simulation, it is sure that these low frequency fluctuations originate from the wind, but it is difficult to discern between incoming flow and the silo wake. Such oscillations typically give rise to quasi-static swaying deformations of the silo, free of resonant effects.

In the following, time averaged results are considered to facilitate comparison of results for one-way and two-way coupled simulations. Figures 14a and b show the time averaged $\bar{E}_{\mathrm{d} j}$ and RMS values $E_{\mathrm{d} j}^{\mathrm{RMS}}$ of the modal deformation energy for the one-way simulation of the single silo for the 50 eigenmodes with the lowest eigenfrequencies. The mean modal deformation energy $\bar{E}_{\mathrm{d} j}$ (figure 14a) gives an indication of the static excitation of the eigenmodes while the RMS values $E_{\mathrm{d} j}^{\mathrm{RMS}}$ (figure 14b) contain information on resonant and quasi-static motion.

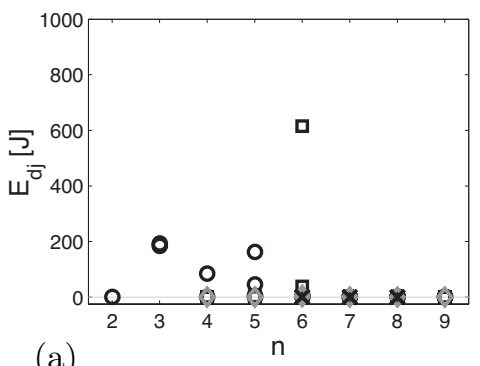

(a)

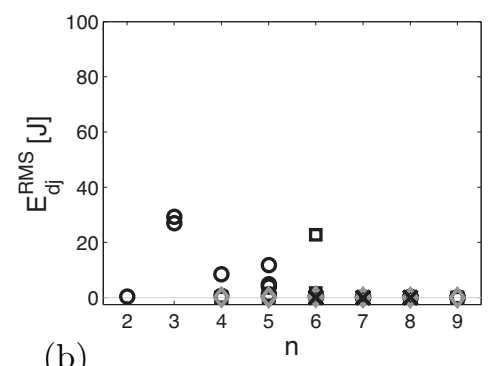

(b)

Figure 14: (left) Mean value and (right) RMS value of the modal contributions to the deformation energy $E_{\mathrm{d} j}(t)$ for the single silo in the one-way simulation. The deformation energy for the lowest 50 eigenmodes is plotted as a function of $n$ while separate mode shapes $(1, n)$ are depicted as a circle $(\circ),(2, n)$ as a square $(\square),(3, n)$ as a diamond $(\diamond)$, and $(4, n)$ as a cross $(\times)$.

The mean modal deformation energies in figure 14a are significantly larger 
than the RMS values in figure $14 \mathrm{~b}$ (shown on a different vertical scale). From these figures, it is observed that modes $\boldsymbol{\Phi}_{2}=(1,3), \boldsymbol{\Phi}_{6}=(1,5)$ and $\boldsymbol{\Phi}_{14}=$ $(2,6)^{*}$ are predominantly excited statically. This is of course related to the aerodynamic forces applied on the structure: high pressures at stagnation, low pressures in the attached boundary layer flows on the sides and a more or less constant pressure after separation. A snapshot of the resulting displacements is shown in figure $15 \mathrm{a}$.

By comparing figures 14a and 14b for the mean and RMS value of the modal deformation energy, respectively, it is clear that structural vibrations are much smaller than the static displacements. According to figure 14b, the mode shapes that are excited dynamically are the eigenmodes with the lowest circumferential wavenumber $n$ and only a half wavelength across the height $(m=1)$. It is noted that the RMS values characterize the magnitude of the dynamic response, regardless of the nature of the latter. For example, the RMS value of mode $\boldsymbol{\Phi}_{14}=(2,6)^{*}$ is due to quasi-static vibration while for modes $\boldsymbol{\Phi}_{2}=(1,3)$ and $\boldsymbol{\Phi}_{6}=(1,5)$ RMS values similar in magnitude correspond to resonant vibrations. This can be observed in figure 13 where the amplitudes of the oscillations at the corresponding eigenfrequencies are smaller for the former than for the latter two.

\section{Two-way simulations}

Two-way simulations are performed for the single silo to assess the change in structural response due to aeroelastic effects and/or interaction between wakeinduced excitation and the vibration. In general, 5 IQN-ILS coupling iterations have to be performed in each time step to ensure equilibrium on the WSI interface. The computational effort required for the two-way simulations is therefore approximately 5 times larger than for the one-way simulations.

The time histories of the modal deformation energy in the one-way (figure 13) and the two-way simulation (figure 16) are compared. Furthermore, the averaged and RMS values of the response in the one-way and two-way simulation are compared (figures 14 and 17). It is observed that the static response and 


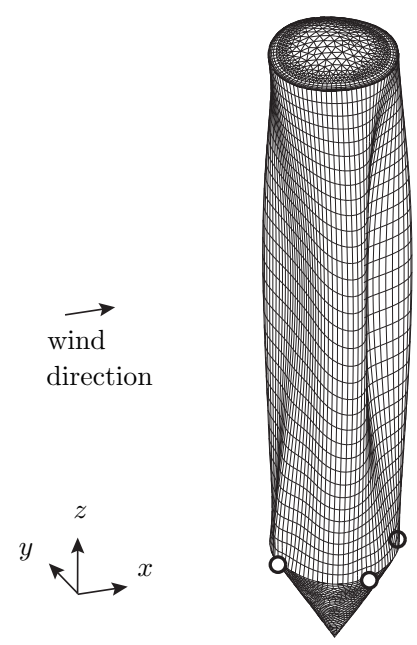

Figure 15: Displacement of the single silo at $t=15 \mathrm{~s}$ in the one-way simulation. The structural displacements are mainly static and are amplified with a scale factor of 40 .

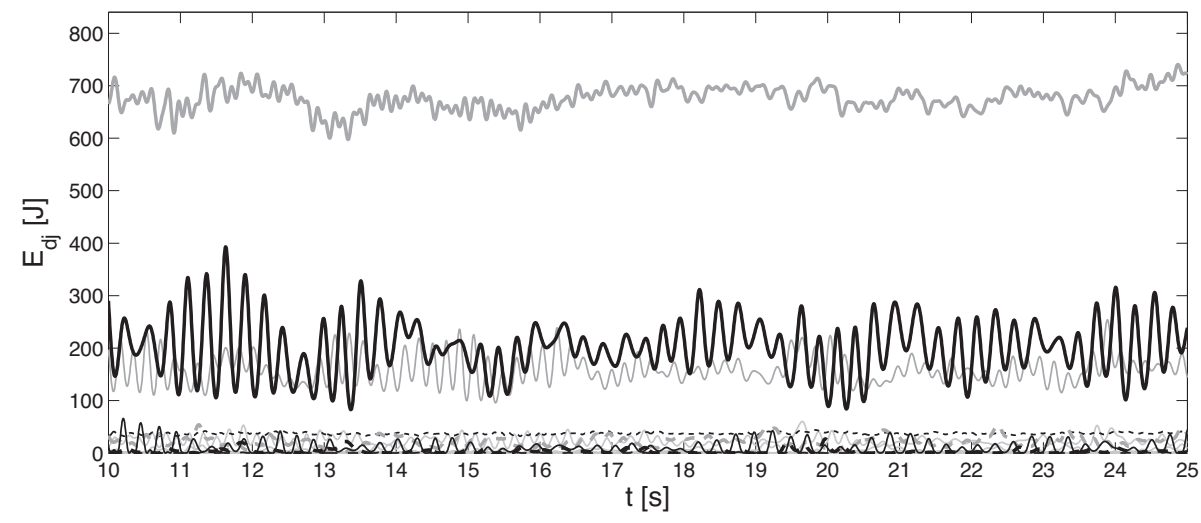

Figure 16: Modal deformation energy $E_{\mathrm{d} j}(t)$ of the first 20 mode shapes, computed from the structural response in the two-way coupled simulation of a single silo: $\boldsymbol{\Phi}_{2}=(1,3)$ (bold black line), $\boldsymbol{\Phi}_{4}=(1,4)$ (thin black line), $\boldsymbol{\Phi}_{6}=(1,5)$ (thin grey line), $\boldsymbol{\Phi}_{14}=(2,6)^{*}$ (bold grey line), $\boldsymbol{\Phi}_{18}=(2,6)$ (dashed black line), and the remaining mode shapes $\boldsymbol{\Phi}_{j}$ (dashed grey lines, with small energy content).

the RMS value of the deformation energy are similar in both simulations. This is reflected in the similar static peak displacements of approximately $0.04 \mathrm{~m}$ in 


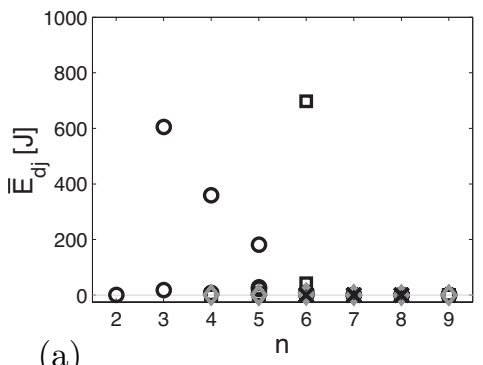

(a)

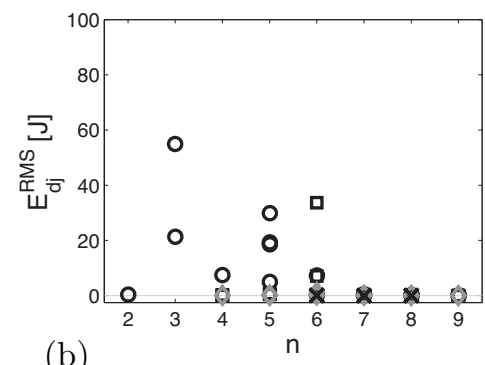

(b)

Figure 17: (left) Mean value and (right) RMS value of the modal contributions to the deformation energy $E_{\mathrm{d} j}(t)$ for the single silo in the two-way simulation. The deformation energy for the lowest 50 eigenmodes is plotted as a function of $n$ while separate mode shapes $(1, n)$ are depicted as a circle $(\circ),(2, n)$ as a square $(\square),(3, n)$ as a diamond $(\diamond)$, and $(4, n)$ as a cross $(x)$.

both simulations. For eigenmode $\boldsymbol{\Phi}_{2}=(1,3)$, the static and RMS value of the dynamic response are distributed differently over the two orthogonal mode shapes in the one-way and two-way simulations but with a similar total value.

In both the one-way and the two-way simulations, the RMS values of the deformation energy are one order of magnitude smaller than the corresponding static values. It is concluded from these observations that qualitatively similar results are found in the one-way and two-way simulation of a single silo, with the same modes contributing to the quasi-static and resonant response of the structure.

\subsection{Silo group simulations}

In order to study the effect of the group arrangement on the wind-induced vibrations, both one-way and two-way simulations have been performed for silos at different locations in the silo group. In the one-way simulations, all corner silos are flexible while in the two-way simulation, only the silo at the windward corner is flexible as this is where the largest vibration amplitudes were observed. The same methodology is applied as in the single silo case. 
One-way simulations

Velocity streamline plots of the flow pattern around the silo group are given in figure 18. It is obvious that the flow pattern around the entire silo group has a much more complex and turbulent nature than that around the single silo. Nevertheless, some trends that were previously discussed are also observed for the group configuration. The formation of a horseshoe vortex is for instance clearly observed (figures 18top and a). The horseshoe vortex is observed at the same location as in Shah and Ferziger (1997) for the wind flow around a cube building with one face perpendicular to the incident wind. In this study, it was found that the primary separation occurs at a saddle point located about one obstacle height ahead of the obstacle. The horseshoe vortex is also much higher than for the single silo configuration, deflecting the approaching wind flow upwards from a larger upstream distance. Separation occurs at the sharp edges at the transverse corners of the building, generating a large wake region with irregular recirculation zones. In the region where the silos are located, a generally similar pattern can be observed but comparing figures $18 \mathrm{~b}$ and c reveals some important differences. Although the flow is only partly attached to the silo structures at the windward side of the silos with a small part of the flow entering the silo array, the global picture remains similar as for the bluff prismatic building where all flow is deflected sideways. At the transverse corners of the silo group (silos 8 and 33), however, flow separation is only partly geometrically triggered and separation occurs on the silo surfaces. At the top of the silo group, the flow is deflected upwards and sideways. It only slightly accelerates but separates from the edges at the top (figures 18top and d). No specific point of reattachment on the top can be observed as for simple block-like geometries in wind flow. This is mainly attributed to the open structure of the silo array with wind flows emerging from the interstitial spaces and interaction with the conical shape of the 40 silo tops. Similarly as for the single silo case, the size of the wake is reduced near the free end of the group but the effect is more limited. The wake behind the silo group is reduced less, creating a larger 
flow resistance as compared to the single silo case.

The influence of the location of a silo in the group on the structural response is investigated by considering the four corner silos: 1, 8, 33 and 40 (figure 2). Additional simulations showed that the response at these locations was representative for all windward and leeward silos in the group.

First, the structural response of silo 1 at the windward corner of the silo group is calculated. The mean and RMS values of the modal deformation energy are shown in figures $19 \mathrm{a}$ and b, respectively. It can be clearly observed that the static deformation of this silo is dominated by eigenmodes $(1,3)$ and $(1,4)$ (figure 19a). Other eigenmodes with low circumferential wavenumbers $(n)$ are also excited, but less pronounced. Roughly the same eigenmodes are also excited dynamically as demonstrated by the RMS values of the modal deformation energy (figure 19b). The RMS values for silo 1 are slightly larger than for the single isolated silo. Note the different vertical scale of the RMS graphs compared to the simulations for a single silo (figure 14).

Predominant static and dynamic excitation of modes $(1,3)$ and $(1,4)$ is also seen in the response snapshots in figure 20. It is observed that the silo wall is deflected inwards due to the large positive pressures at the windward side. When moving downstream along the silo wall, the attached boundary layer causes suction and the shell is deformed outwards. The largest structural displacements, however, are found in the small gaps between two adjacent silos. Due to the larger wind velocities in these narrow passages, negative surface pressures develop and the silos deform accordingly. The magnitude of the structural displacements is as high as $0.07 \mathrm{~m}$ in these interstitial spaces. These displacement values are large in comparison with the total distance of only $0.3 \mathrm{~m}$ between two neighbouring silos. The vibration amplitudes, however, are significantly smaller (approx. 0.01 to $0.02 \mathrm{~m}$ ).

For the silos on the transverse corners of the silo group, i.e. silos 8 and 33 , the results of the one-way simulations are shown in figures $19 \mathrm{c}$ to f. For silos 8 and 33 , the RMS value of the modal contributions is significantly larger than for a single silo or silo 1 in the group. This is in agreement with the time history of 


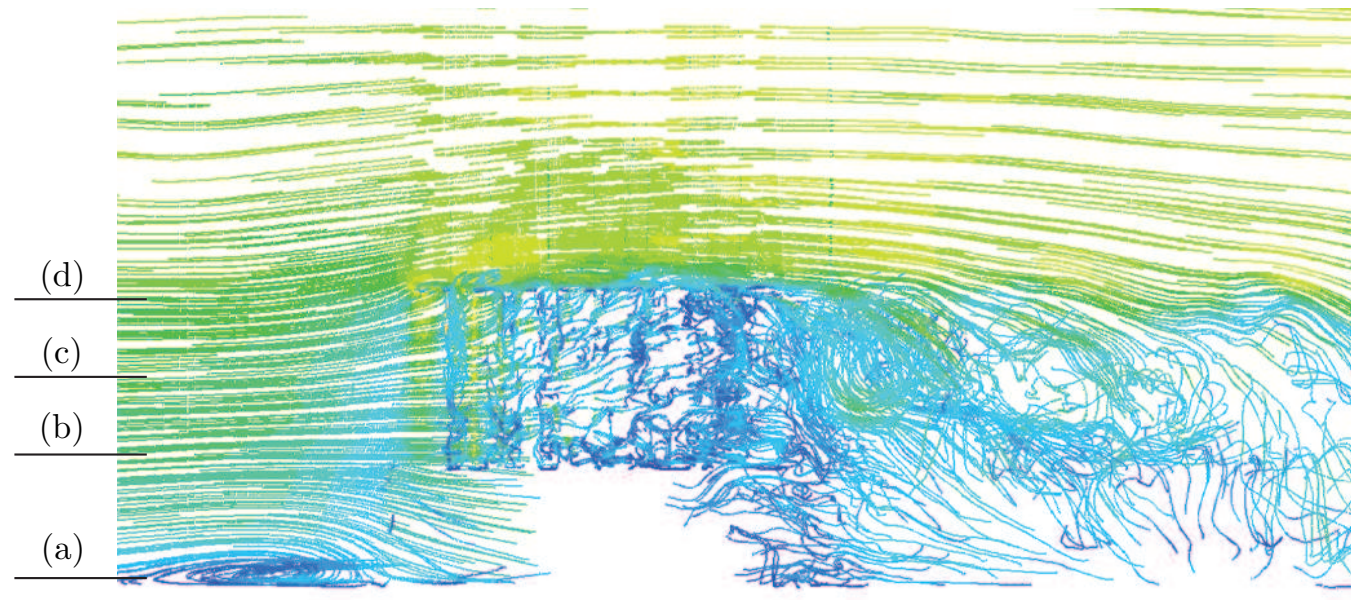

(a)

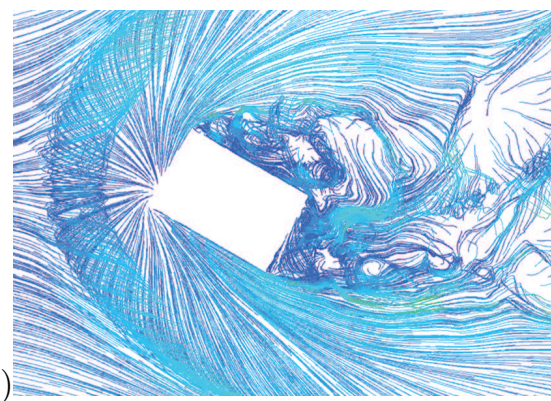

(c)

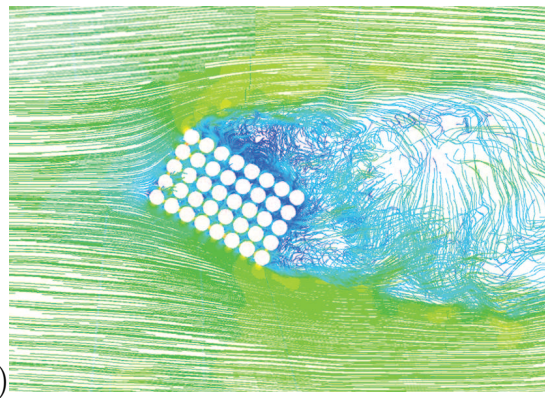

(b)

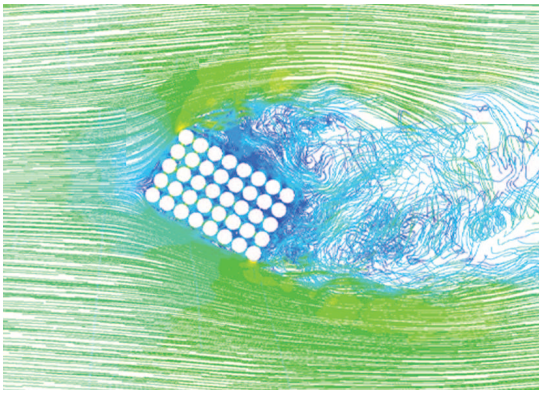

(d)

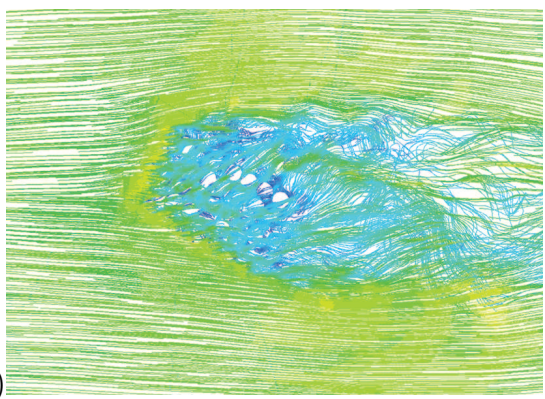

Figure 18: Streamlines of the wind flow around the silo group after $40 \mathrm{~s}$, colored according to the velocity magnitude, released from (top) a vertical plane $y=0 \mathrm{~m}$ and horizontal planes at (a) $z=0.5 \mathrm{~m}$, (b) $z=17.16 \mathrm{~m}$, (c) $z=29.16 \mathrm{~m}$, and (d) $z=41.16 \mathrm{~m}$. 
(a)
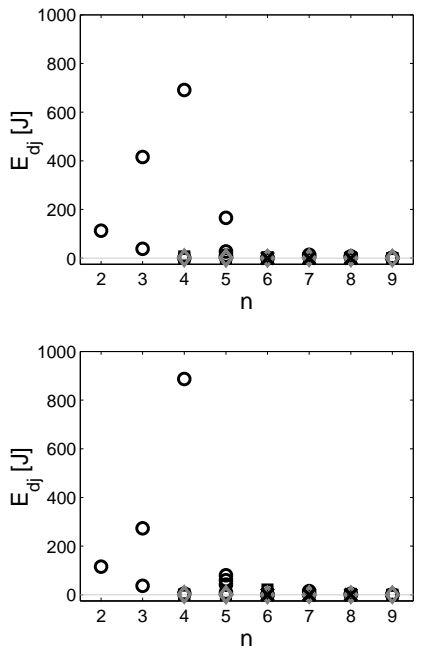

(c)

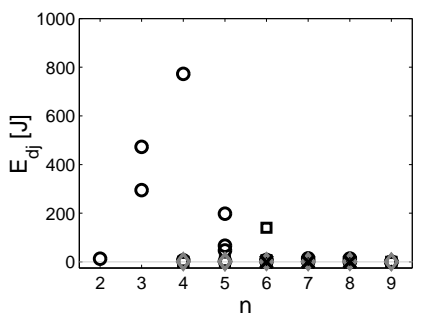

(e)

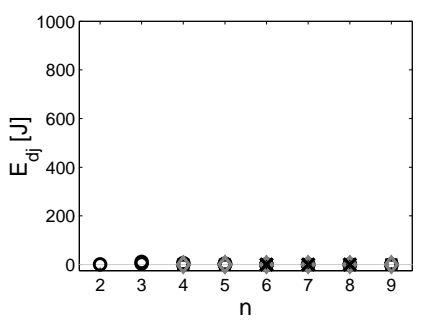

(b)
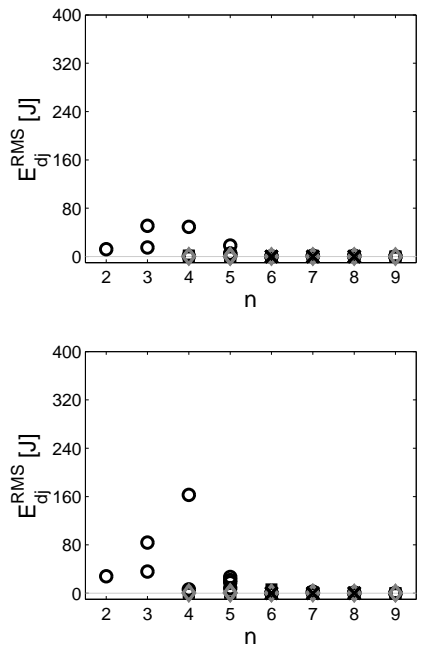

(d)

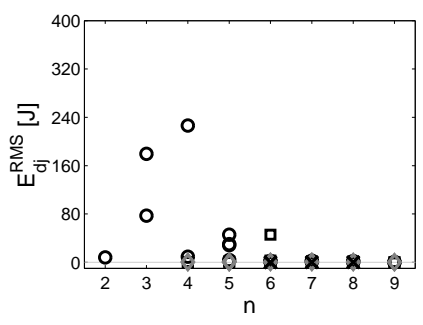

(f)

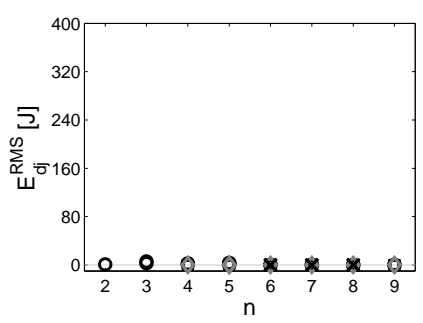

Figure 19: (left) Mean value and (right) RMS value of the modal contributions to the deformation energy $E_{\mathrm{d} j}(t)$ in the one-way simulation of the silo group. The first row shows silo 1 , the second row silo 8 , the third row silo 33 and the last row silo 40 . The deformation energy for the lowest 50 eigenmodes is plotted as a function of $n$ while separate mode shapes $(1, n)$ are depicted as a circle $(\circ),(2, n)$ as a square $(\square),(3, n)$ as a diamond $(\diamond)$, and $(4, n)$ as a cross $(\times)$.

the drag coefficients for these silos in figure 9. It is interesting that also at these locations eigenmodes $(1,3)$ and $(1,4)$ are predominantly excited, both statically 


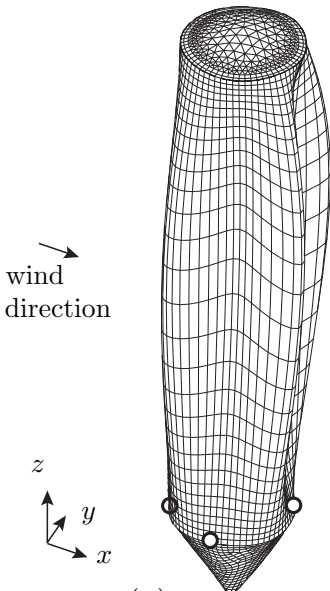

(a)

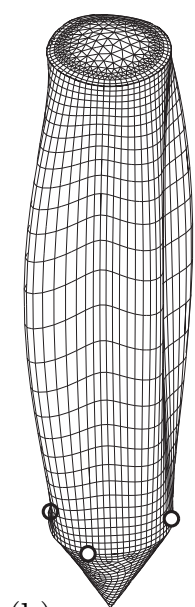

(b)

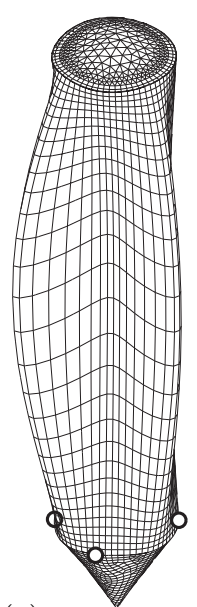

(c)

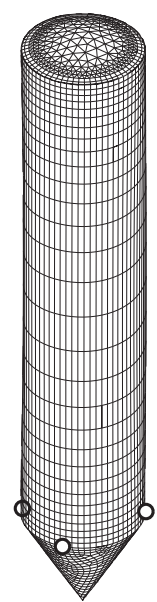

(d)

Figure 20: Displacement at $t=10 \mathrm{~s}$ of (a) silo 1, (b) silo 8, (c) silo 33, and (d) silo 40 of the one-way simulation of the silo group. The structural displacements are mainly static and are amplified with a scale factor of 40 .

and dynamically. This is related to the fact that these modes have the lowest eigenfrequencies and are hence most susceptible to the low frequency content of the turbulent wind excitation. Furthermore, the vicinity of the neighbouring silos in a square arrangement and the resulting high wind velocities that are developed in the gaps between two adjacent silos automatically gives rise to the excitation of mode $(1,4)$. Despite the differences in the RMS values of the modal contributions to the deformation energy between silos 8 and 33 on the one hand and silo 1 on the other hand, it should be noted that the displacements found on corner silos 8 and 33 are of the same order of magnitude as for silo 1 at the windward side, as illustrated in figure 20, mainly due to the dominance of the mean value in the deformation energy.

Finally, the results of the one-way simulation for the lee side corner silo 40 are shown in figures $19 \mathrm{~g}$ and $\mathrm{h}$. As opposed to the other corner silos, the modal deformation energy for this silo is negligibly small for all eigenmodes. The remarkable difference between the structural response at the lee side corner 
(silo 40) and the other three corner silos (silos 1, 8 and 33) of the group is visualized in figure 20 . The radial static displacements are only approximately $0.01 \mathrm{~m}$ while vibration amplitudes are negligibly small for silo 40 .

Overall, the patterns of the predicted vibration correspond well with the observed ovalling vibrations in the Antwerp silo group during the 2002 storm. Vibrations are observed at the windward side of the group and the modes that are preferentially excited seem to be those giving rise to the observed ovalling motion. In these one-way simulations, however, the magnitude of the vibration levels (approx. 0.01 to $0.02 \mathrm{~m}$ ) is still much smaller than the ones observed (approx. $0.1 \mathrm{~m}$ ) in October 2002.

\section{Two-way simulations}

Although the one-way simulations indicated which silos were subjected to ovalling vibrations, the resulting vibration amplitudes are smaller than observed. Therefore, a fully coupled WSI simulation is performed for the group configuration, taking into account aeroelastic effects and/or interactions between the wake-induced excitation and the vibration. In order to obtain results within a reasonable computation time, only a single flexible silo at the windward side of the group (silo 1) is considered while all other silos in the group are rigid.

At the start of the two-way simulation, a direct transfer of the large structural displacements to the flow field inevitably leads to a long and significant transition in the fluid and structural response. The corresponding amplitudes of the vibrations are so large that the flexible silo collides with its neighbouring rigid silos due to the narrow gaps. To solve this issue, the aerodynamic forces that are passed from the flow solver to the structural solver are gradually increased during a limited period of time $(5 \mathrm{~s})$.

In general, approximately 5 IQN-ILS coupling iterations are required per time step. As a result, simulating $1 \mathrm{~s}$ of wind flow in this two-way simulation of the silo group requires about 70 hours of computing time.

Figure 21 depicts the modal deformation energy as a function of time for the two-way simulation of silo 1 . The initial part of the simulation until $t=5 \mathrm{~s}$ 
where the solution gradually evolves to the stationary regime should obviously be disregarded but from $t=5 \mathrm{~s}$ on the response of the structure to the full aerodynamic forces is obtained. The fluctuations in the deformation energy reach far beyond the corresponding levels in any of the one-way simulations. At approximately $t=8 \mathrm{~s}$ a peak is reached and the structural response decreases before rising again at approximately $t=12 \mathrm{~s}$. This two-way simulation shows that WSI simulations are recommended to investigate ovalling vibrations in silo groups.

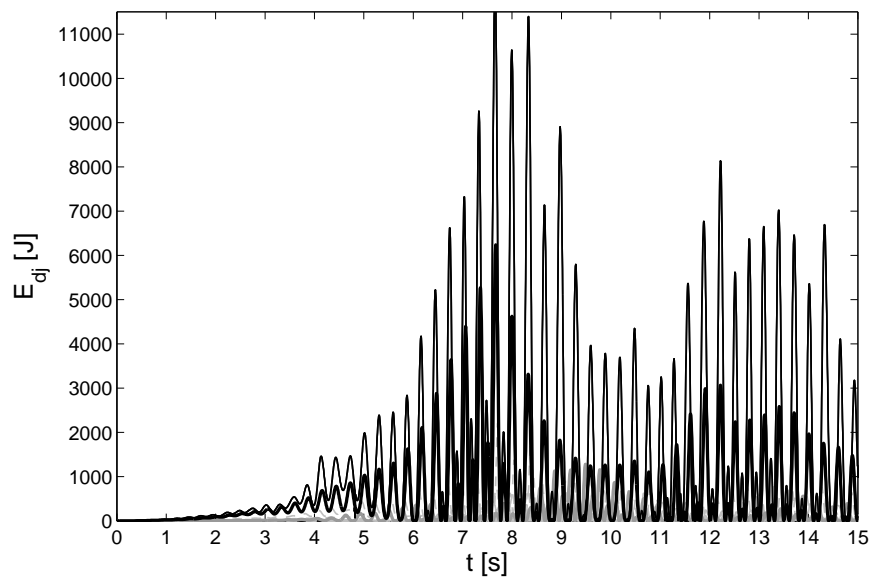

Figure 21: Modal deformation energy $E_{\mathrm{d} j}(t)$ for the first 20 mode shapes, based on the structural response of the upwind silo 1 in the two-way simulation of the entire silo group: $\boldsymbol{\Phi}_{1}=(1,3)$ (bold grey line), $\boldsymbol{\Phi}_{2}=(1,3)$ (bold black line), $\boldsymbol{\Phi}_{4}=(1,4)$ (thin black line), and the remaining mode shapes $\boldsymbol{\Phi}_{j}$ (dashed grey lines, with small energy content).

Taking into account the difference in vertical scale in figures $22 \mathrm{a}$ and $\mathrm{b}$ and $19 \mathrm{a}$ and $\mathrm{b}$, it is observed that the result of the two-way simulation of silo 1 (figures 22a and b) is distinctly different from the one in the one-way simulation (figures 19a and b). As opposed to the one-way simulations, the mean value and the RMS value of the modal contributions to the deformation energy have the same order of magnitude in the two-way simulations. The modal deformation energy graphs indicate a marked aeroelastic effect and/or interaction between the wake-induced excitation and the vibration in the structural response as a 
result of changing wind flow patterns in vicinity of the silo structure. To distinguish an aeroelastic effect from interaction between the wake-induced excitation and the vibration, the calculations would have to be repeated for higher wind flow velocities. Aeroelastic effects would have to remain present above the critical velocity, whereas interaction between the wake-induced excitation and the vibration is expected to disappear when the wind velocity exceeds the resonant peak. However, repeating the analysis for different wind speeds is beyond the scope of this work. Hence, no statements on aeroelastic effect versus interaction between the wake-induced excitation and the vibration can be made based on the present study. In a study by Païdoussis et al. (1988) ovalling was identified as an aeroelastic flutter phenomenon using a model with two-dimensional flow around a single shell that is supported at both ends. As the silo group is only fixed at one end and the flow around it has been shown to be three-dimensional, it is not certain whether this conclusion by Païdoussis et al. (1988) holds for this silo group as well.

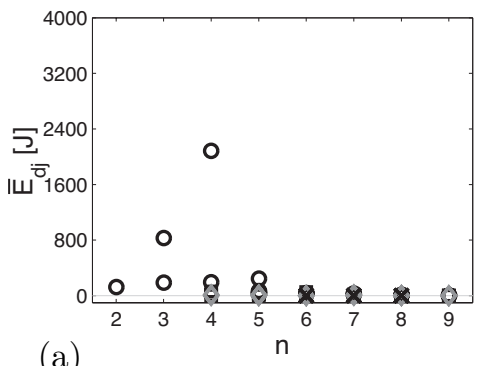

(a)

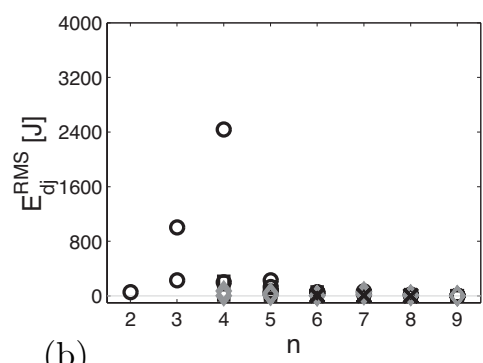

(b)

Figure 22: (a) Mean value and (b) RMS value of the modal contributions to the deformation energy $E_{\mathrm{d} j}(t)$ for silo 1 in the two-way simulation of the silo group. The deformation energy for the lowest 50 eigenmodes is plotted as a function of $n$ while separate mode shapes $(1, n)$ are depicted as a circle $(\circ),(2, n)$ as a square $(\square),(3, n)$ as a diamond $(\diamond)$, and $(4, n)$ as a $\operatorname{cross}(x)$.

To assess the magnitude of the structural vibrations in the two-way simulation, the time history of the radial displacements at two discrete points (A and $\mathrm{B}$ in figure 23) along the circumference and at mid-height of silo 1 is shown in 
figure 24 with positive values indicating outward displacements. In the small gap between silos 1 and 9 (point A, figure 24a), the vibration amplitudes increase up to about $0.05 \mathrm{~m}$. At the lee side of the silo (point B, figure 24b) the increase in the amplitude is slightly smaller but still very distinct. The vibrations in this simulation are significantly larger than in the one-way simulations and tend to the observed vibration amplitudes during the storm in 2002 (order of magnitude of $0.1 \mathrm{~m}$ ). Furthermore, the dominant excitation of eigenmodes $(1,3)$ and $(1,4)$ is in line with observations.

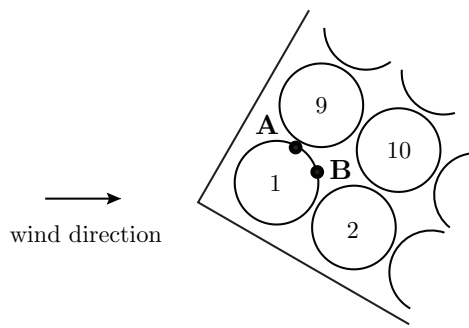

Figure 23: Location of two discrete points A and B along the circumference and at mid-height $\left(z_{\mathrm{s}}=12.5 \mathrm{~m}\right)$ of silo 1 in the group arrangement.

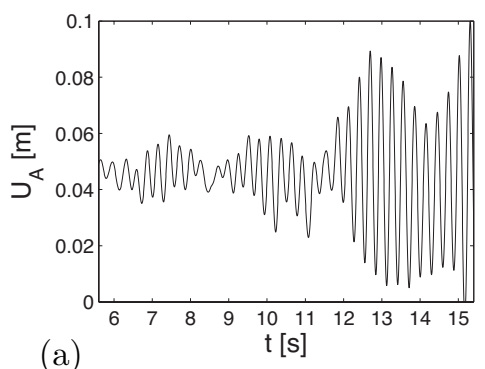

(a)

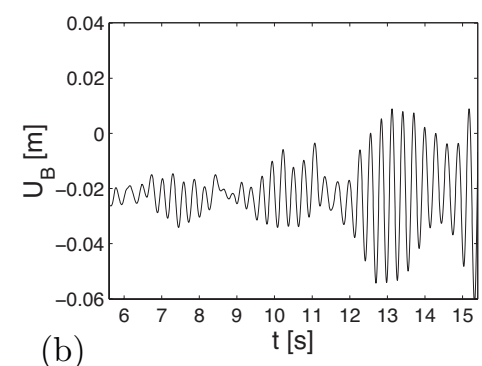

(b)

Figure 24: Radial displacements at two discrete points A and B (cfr. figure 23) along the circumference and at mid-height of silo 1 in the two-way simulation of the group arrangement. 


\section{Conclusions}

The comparison between the one-way and two-way simulations did not reveal significant differences for the single silo case. By contrast, the vibration amplitudes for the silo group are significantly larger in the two-way simulations than in the one-way simulations. As the importance of two-way simulations is difficult to estimate a priori, it is thus recommended to take the structural displacement into account in the wind flow simulation for WSI simulations of similar structures.

It is also concluded that the average and/or the RMS value of the wind loading on the windward side of a silo group can be much larger than on a single silo. This observed group effect is in agreement with the outcome of the Ferrybridge investigation, but appears on the windward side of closely spaced silos as opposed to the leeward side of more widely spaced cooling towers. Furthermore, comparing the two-way simulations for the single silo with the two-way simulations for the silo group demonstrates that the vibration amplitudes are significantly larger for the group. So, both the Ferrybridge investigation and this study indicate that objects in group should not be designed using wind loading data or norms for a single object.

Finally, the two-way simulations of the silo group show results in agreement with the observations during the storm. Extensive analysis of the grid size and time step size gives an idea of the uncertainty on the results. However, the duration of the simulations mentioned throughout this article and the required computing power limit the use of the proposed modelling as engineering tool to special investigations at present.

\section{Acknowledgements}

The research in this paper has been performed within the frame of the FWO project G.0275.08 "Efficient analysis of fluid-structure interaction problems in structural dynamics". This work was carried out using the STEVIN Supercomputer Infrastructure at Ghent University, funded by Ghent University, the 
Flemish Supercomputer Center (VSC), the Hercules Foundation and the Flemish Government (department EWI).

\section{References}

Abaqus 6.10, 2010. User's Manual. Dassault Systèmes Simulia Corp.

AIJ, 2006. Recommendations for Loads on Buildings. Architectural Institute of Japan.

ANSI, 2010. ASCE 7-10: Minimum Design Loads for Buildings and Other Structures. American National Standards Institute.

Batina, J., 1990. Unsteady Euler airfoil solutions using unstructured dynamic meshes. AIAA Journal 28 (8), 1381-1388.

Bazilevs, Y., Hsu, M.-C., Kiendl, J., Wüchner, R., Bletzinger, K.-U., 2011. 3D simulation of wind turbine rotors at full scale. Part II: Fluid-structure interaction modeling with composite blades. International Journal for Numerical Methods in Fluids 65, 236-253.

Belloli, M., Collina, A., Rossa, L., Squicciarini, G., July 2011. Wind tunnel tests on different erection stages of a cable stayed bridge. In: De Roeck, G., Degrande, G., Lombaert, G., Müller, G. (Eds.), Proceedings of the 8th International Conference on Structural Dynamics, EURODYN 2011. Leuven, Belgium, pp. 1325-1332.

Billah, K., Scanlan, R., 1991. Resonance, Tacoma Narrows bridge failure, and undergraduate physics textbooks. American Journal of Physics 59 (2), 118124.

BIN, December 2010. NBN EN 1991-1-4:2005/AC Eurocode 1: Actions on structures - Part 1-4: General actions - Wind actions (+ AC:2010). Belgisch Instituut voor Normalisatie. 
Blevins, R., 1990. Flow-Induced Vibration, 2nd Edition. Van Nostrand Reinhold.

Blocken, B., Carmeliet, J., 2006. The influence of the wind-blocking effect by a building on its wind-driven rain exposure. Journal of Wind Engineering and Industrial Aerodynamics 94 (2), 101-127.

Castro, I., 2003. CFD for external aerodynamics in the built environment. The QNET-CFD Network Newsletter 2 (2), 4-8.

COST Action 732, 2007. "Quality assurance and improvement of microscale meteorological models". In: Franke, J., Hellsten, A., Schlünzen, H., Carissimo, B. (Eds.), Best practice guideline for the CFD simulation of flows in the urban environment. Office for Official Publications of the European Communities, Luxembourg, pp. 1-52.

COST Action C14, 2004. "Impact of wind and storms on city life and built environment". working group 2: CFD techniques. In: Franke, J., Hirsch, C., Jensen, A., Krüs, H., Schatzmann, M., Westbury, P., Miles, S., Wisse, J., Wright, N. (Eds.), Recommendations on the use of CFD in predicting pedestrian wind environment. Office for Official Publications of the European Communities, Luxembourg, pp. 1-28.

Degroote, J., 2013. Partitioned simulation of fluid-structure interaction. Archives of Computational Methods in Engineering 20, 185-238.

Degroote, J., Bathe, K.-J., Vierendeels, J., 2009. Performance of a new partitioned procedure versus a monolithic procedure in fluid-structure interaction. Computers and Structures 87, 793-801.

Donea, J., Guiliani, S., Halleux, J., 1982. An arbitrary Lagrangian-Eulerian finite-element method for transient dynamic fluid structure interactions. Computer Methods in Applied Mechanics and Engineering 33 (1-3), 689-723.

Donea, J., Huerta, A., Ponthot, J.-P., Rodríguez-Ferran, 2004. Arbitrary Lagrangian-Eulerian methods. In: Stein, E., de Borst, R., Hughes, T. (Eds.), 
Encyclopedia of Computational Mechanics. Volume 1: Fundamentals. John Wiley \& Sons, pp. 413-438.

Dooms, D., Degrande, G., De Roeck, G., Reynders, E., 2006. Finite element modelling of a silo based on experimental modal analysis. Engineering Structures $28(4), 532-542$.

Fluent 14.5, 2012. User's Guide. Ansys Inc.

Gopalan, H., Jaiman, R., 2015. Numerical study of the flow interference between tandem cylinders employing non-linear hybrid URANS-LES methods. Journal of Wind Engineering and Industrial Aerodynamics 142, 111-129.

Gorski, J., Mikulski, T., Ozieblo, M., Winkemann, K., 2015. Effect of geometric imperfections on aluminium silo capacities. Stahlbau 84 (1), 52-57.

Hillewaere, J., Dooms, D., Van Quekelberghe, B., Degroote, J., Vierendeels, J., De Roeck, G., G. Degrande, G., 2012. Unsteady Reynolds averaged NavierStokes simulation of the post-critical flow around a closely spaced group of silos. Journal of Fluids and Structures 30, 51-72.

Hojjat, M., Stavropoulou, E., Gallinger, T., Israel, U., Wüchner, R., Bletzinger, K.-U., 2010. Fluid-structure interaction in the context of shape optimization and computational wind engineering. In: Bungartz, H.-J., Mehl, M., Schäfer, M. (Eds.), Fluid-Structure Interaction II. Modelling, Simulation, Optimization. Vol. 73 of Lecture Notes in Computational Science and Engineering. Springer, pp. 351-381.

Holmes, J., 2015. Wind Loading of Structures, 3rd Edition. Taylor \& Francis.

Löhner, R., Yang, C., 1996. Improved ALE mesh velocities for moving boundaries. Communications in Numerical Methods in Engineering 12 (10), 599608.

Mannini, C., Sbragi, G., Schewe, G., Borri, C., July 2011a. Determination of flutter derivatives for a box-girder bridge deck through URANS simulations. 
In: De Roeck, G., Degrande, G., Lombaert, G., Müller, G. (Eds.), Proceedings of the 8th International Conference on Structural Dynamics, EURODYN2011. Leuven, Belgium, pp. 1473-1480.

Mannini, C., Soda, A., Schewe, G., 2011b. Numerical investigation on the threedimensional unsteady flow past a 5:1 rectangular cylinder. Journal of Wind Engineering and Industrial Aerodynamics 99 (4), 469-482.

Menter, F., Kuntz, M., Langtry, R., October 2003. Ten Years of Industrial Experience with the SST Turbulence Model. In: Hanjalić, K., Nagano, Y., Tummers, M. (Eds.), Proceedings of the Fourth International Symposium on Turbulence, Heat and Mass Transfer. Begell House, Inc., Antalya, Turkey, pp. 625-632.

Michalski, A., Kermel, P., Haug, E., Löhner, R., Wüchner, R., Bletzinger, K.-U., 2011. Validation of the computational fluid-structure interaction simulation at real-scale tests of a flexible $29 \mathrm{~m}$ umbrella in natural wind flow. Journal of Wind Engineering and Industrial Aerodynamics 99, 400-413.

Païdoussis, M., Price, S., Ang, S.-Y., 1988. Ovalling oscillations of cylindrical shells in cross flow: A review and some new results. Journal of Fluids and Structures 2 (1), 95-112.

Païdoussis, M., Price, S., de Langre, E., 2011. Fluid-Structure Interactions: Cross-Flow-Induced Instabilities. Cambridge University Press.

Païdoussis, M., Wong, D.-M., 1982. Flutter of thin cylindrical shells in cross flow. Journal of Fluid Mechanics 115, 411-426.

Park, C.-W., Lee, S.-J., 2004. Effects of free-end corner shape on flow structure around a finite cylinder. Journal of Fluids and Structures 19, 141-158.

Patankar, S., Spalding, D., 1972. A calculation procedure for heat, mass and momentum transfer in three-dimensional parabolic flows. International Journal of Heat and Mass Transfer 15 (10), 1787-1806. 
Pope, R., 1994. Structural deficiencies of natural draught cooling towers at UK power stations part 1: failures at Ferrybridge and Fiddlers Ferry. Proceedings of the Institution of Civil Engineers-Structures and Buildings 104 (1), 1-10.

Price, S., Païdoussis, M., Macdonald, R., Mark, B., 1987. The flow-induced vibration of a single flexible cylinder in a rotated square array of rigid cylinders with a pitch-to-diameter ratio of 2.12. Journal of Fluids and Structures 1 (3), $359-378$.

Quinn, A., Wilson, M., Reynolds, A., Couling, S., Hoxey, R., 2001. Modelling the dispersion of aerial pollutants from agricultural buildings - An evaluation of computational fluid dynamics (CFD). Computers and Electronics in Agriculture 30, 219-235.

Shah, K., Ferziger, J., 1997. A fluid mechanicians view of wind engineering: Large eddy simulation of flow pas a cubic obstacle. Journal of Wind Engineering and Industrial Aerodynamics 67-68, 211-224.

Shellard, H., 1967. Collapse of cooling towers in a gale, Ferrybridge, 1 November 1965. Weather 22 (6), 232-240.

Smirnov, A., Shi, S., Celik, I., 2001. Random flow generation technique for large eddy simulations and particle-dynamics modeling. Journal of Fluids Engineering, Transactions of the ASME 123, 359-371.

Spalart, P., Deck, S., Shur, M., Squires, K., Strelets, M., Travin, A., 2006. A new version of detached-eddy simulation, resistant to ambiguous grid densities. Theoretical and Computational Fluid Dynamics 20 (3), 181-195.

Squires, K., Krishnan, V., Forsythe, J., 2008. Prediction of the flow over a circular cylinder at high reynolds number using detached-eddy simulation. Journal of Wind Engineering and Industrial Aerodynamics 96 (10-11), 15281536.

Tominaga, Y., Mochida, A., Yoshie, R., Kataoka, H., Nozu, T., Yoshikawa, M., Shirisawa, T., 2008. AIJ guidelines for practical applications of CFD to 
pedestrian wind environment around buildings. Journal of Wind Engineering and Industrial Aerodynamics 96, 1749-1761.

Uernatsu, Y., Yasunaga, J., Koo, C., 2015. Design wind loads for open-topped storage tanks in various arrangements. Journal of Wind Engineering and Industrial Aerodynamics 138, 77-86.

VDI-Richtlinie 3783, 2003. Blatt 9 (Entwurf): Umweltmeteorologie. Prognostische Mikroskalige Windfeldmodelle. Evaluierung für Gebäude- und Hindernisumströmung. Verrein Deutscher Ingenieure.

Šarkić, A., Fisch, R., Höffer, R., Bletzinger, K.-U., 2012. Bridge flutter derivatives based on computed, validated pressure fields. Journal of Wind Engineering and Industrial Aerodynamics 104-106, 141-151.

Žukauskas, A., Ulinskas, R., Katinas, V., Karni, J., 1988. Fluid Dynamics and Flow-Induced Vibrations of Tube Banks. Hemisphere Publishing Corporation.

Weaver, D., Lian, H., Huang, X., 1993. Vortex shedding in rotated square arrays. Journal of Fluids and Structures 7 (2), 107-121.

Zdravkovich, M., 1997. Flow Around Circular Cylinders, Volume 1: Fundamentals. Oxford University Press.

Zhang, C., 1994. Numerical prediction of turbulent recirculating flows with a $k-\varepsilon$ model. Journal of Wind Engineering and Industrial Aerodynamics 51, $177-201$.

Zhao, Y., Cao, Q.-S., Su, L., 2013. Buckling design of large circular steel silos subject to wind pressure. Thin-Walled Structures 73, 337-349.

Zhao, Y., Lin, Y., Shen, Y.-B., 2014. Wind loads on large cylindrical opentopped tanks in group. Thin-Walled Structures 78, 108-120. 